Article

\title{
The Physical Density of the City-Deconstruction of the Delusive Density Measure with Evidence from Two European Megacities
}

\author{
Hannes Taubenböck*, Ines Standfuß, Martin Klotz and Michael Wurm \\ Taubenböck, German Aerospace Center (DLR), German Remote Sensing Data Center (DFD), Oberpfaffenhofen, \\ Wessling 82234, Germany; ines.standfuss@dlr.de (I.S.); martin.klotz@dlr.de (M.K.); michael.wurm@dlr.de (M.W.) \\ * Correspondence: hannes.taubenboeck@dlr.de
}

Academic Editors: Jamal Jokar Arsanjani and Wolfgang Kainz

Received: 6 June 2016; Accepted: 4 November 2016; Published: 9 November 2016

\begin{abstract}
Density is among the most important descriptive as well as normative measures in urban research. While its basic concept is generally understandable, approaches towards the density measure are manifold, diverse and of multidimensional complexity. This evolves from differing thematic, spatial and calculative specifications. Consequently, applied density measures are often used in a subjective, non-transparent, unspecific and thus non-comparable manner. In this paper, we aim at a systematic deconstruction of the measure density. Varying thematic, spatial and calculative dimensions show significant influence on the measure. With both quantitative and qualitative techniques of evaluation, we assess the particular influences on the measure density. To do so, we reduce our experiment setting to a mere physical perspective; that is, the quantitative measures building density, degree of soil sealing, floor space density and, more specifically, the density of generic structural classes such as open spaces and highest built-up density areas. Using up-to-date geodata derived from remote sensing and volunteered geographic information, we build upon high-quality spatial information products such as 3-D city models. Exemplified for the comparison of two European megacities, namely Paris and London, we reveal and systemize necessary variables to be clearly defined for meaningful conclusions using the density measure.
\end{abstract}

Keywords: building density; soil sealing; floor space density; open spaces; 3-D model; structural classes; remote sensing; volunteered geographic information; comparative urban research

\section{Introduction}

Density is one of the most crucial characteristics of urbanity (e.g., [1]). Walking through London's City district, this becomes immanently perceptible through diverse simultaneous observations: masses of people and vehicles moving vibrantly through the complex arrangement of space; through close-knit streets, alongside a mix of building types with different functionalities, passing open spaces within a comparatively small area. No doubt, this typical urban scenery implies density, the embodiment of city life.

The term density is popular as it is generally understandable and seems to be self-explanatory. Burdett et al. [2] found that a majority of people naturally understand the concept of urban density. Beyond its natural perception, its scientific rationale, its simple mathematical derivation, its apparent comparability and its possibility for verification put it among the most important descriptive, explanatory as well as normative measures in urban research [3]. However, the density measure is delusive; there is a lack of clarity about how to measure and how to use density within the scientific debate [4,5]. Consequently, its application often lacks clearness and consistency that derives from its multidimensional nature, i.e., from different thematic (population, jobs, buildings, etc.) and spatial 
dimensions (administrative boundaries, postcode areas, districts, blocks, etc.) as well as from the application of different aggregation functions (gross vs. net densities, etc.).

We exemplify the delusiveness of the density measure using a classical thematic variable in geography-population: Based on 2011 census data [6], the megacity Paris has a population density of 21,258 people per $\mathrm{km}^{2}$. London's population density is, in contrast, only 5432 people per $\mathrm{km}^{2}$. While such contrasting realities are often used for comparing cities, in this case their usage neglects that these data are de-facto not comparable: the Paris example refers to an area of $105.4 \mathrm{~km}^{2}$, while the London case refers to a significantly larger reference area of $1572 \mathrm{~km}^{2}$, an issue commonly known as the modifiable areal unit problem (MAUP) [7]. Consequently, the arbitrariness of the two reference units affects results and triggers misleading interpretations with ineligible applications of the density measure. In this case, the inherent delineation issues that arise from the employment of artificial administrative city boundaries limit the density measure to a simple rhetorical medium that is prone to misconception. Such conceptual issues implicitly introduce the complexity that comes along with its usage and explain why an internationally accepted standard for the density measure is yet inexistent $[5,8]$.

We believe a better understanding of the various conceptual and empirical aspects of the density measure is of crucial importance for the scientific debate; especially as the measure has evolved-beyond demographics-to a sort of self-referential system that is frequently employed in a multitude of disciplines, including geography (e.g., [9,10]), planning (e.g., [11,12]), economics (e.g., [13,14]), social science (e.g., [15,16]), or politics (e.g., [2]), among others. All these thematic aspects feed the complex interplay of flows of information, flows of resources, and political regulations which are all together underpinning the spatial organization and structure of cities hitherto [17]. Density is one of the most commonly used measures to quantify these thematic aspects in space. The focus of such works concentrates on density gradients or patterns relative to urban cores [18] analyzing patterns of specific subsystems such as population, jobs, economic productivity, amenities, or traffic, among others (an overview on commonly applied density indicators and their meaning is presented by [19]); in any of these studies intrinsically the built urban environment is addressed. Indicators applied to explicitly describe the physical urban structures are for instance the street network density (e.g., [20]), density of impervious surfaces (e.g., [21]), building density (e.g., [22]) or related parameters such as the floor space density—as a measure for 3D building density (e.g., [23]). Analytical approaches on the physical density of cities use, e.g. gradient analysis (e.g., [24-26]), exploratory approaches (e.g., [27-30]) or spatial metrics (e.g., [31-33]). In these studies, the density measure is used as a descriptive, empirical variable for physical urban structure as well as an explanatory variable for issues such as energy consumption, commuting times, work patterns, etc. However, an agreed definition on how to address physical urban morphology or urban form is inexistent [34]. Thus, empirical studies on physical density of a city are fraught with challenges that are conceptual, as clearness on aggregation functions, spatial or thematic dimensions is mostly absent $([4,35,36])$. Beyond, empirical studies on physical density are fraught with restrictions on data availability. Consequently, density studies are mostly carried out for individual cities or few case studies with a conceptual vagueness that impedes the demand for international comparative density analyses.

In this study, we aim to add to the literature by deconstructing the (conceptual and empirical) delusiveness of the density measure. By considering multiple aspects of the density concept (from aggregation functions to spatial and thematic dimensions) a framework is set that systemizes the variables to be considered when applying density measures. Based on this framework, we systematically analyze the influence that particular thematic, spatial or calculative concepts have on results; with it, we reveal that disregarding these aspects inevitably leads to misleading, inappropriate or simply incorrect usages and interpretations of the density measure.

The multitude of thematic density dimensions-as shown above-is inexhaustible; to reduce complexity we focus on a mere physical perspective, i.e., variables that describe the physical arrangement of the built environment; these are the established quantitative measures building density, 
degree of soil sealing, floor space density and, more specifically, the density of generic structural classes such as open spaces and highest built-up density areas. Hence, we argue that this family of variables and the respective analyses (using various spatial dimensions and calculation techniques) can be seen as a blueprint to deconstruct the delusiveness of the measure and, by this, allows highlighting the fragility of the concept. In turn, this family of variables constructs a framework directing future studies to meaningful applications and conclusions using the density measure.

The remainder of the work is structured as follows: Section 2 briefly presents the two cities studied in this work; in addition, the spatial data sources are introduced. In Section 3, the conceptual framework is presented introducing the thematic and spatial dimensions of the density measure along with the respective calculus. In addition meaningful evaluation techniques to deconstruct physical density are shown. The quantitative and qualitative evaluations of the density measure for the studied cities are presented in Section 4 followed by their discussion in Section 5 . Section 6 concludes with a final perspective.

\section{Study Sites and Data}

\subsection{Study Sites}

Two European cities, Paris, France and London, UK, are selected as study sites. We choose these two cities because they are comparable from geographic, demographic and organizational perspectives. Located just $340 \mathrm{~km}$ apart, both cities are capitals of their countries, and are, with 12.34 (Paris) and 13.61 (London) million people, as per definition, megacities [37].

\subsection{Spatial Data Sets}

For a systematic deconstruction of the physical density for the two European megacities, we employ available state-of-the-art geo-information products derived from original remote sensing data, standardized Earth observation layers and volunteered geographic information (VGI).

1. 3-D city models: For London, we use the UKMap building inventory which provides information on building footprints and their respective height. Height and footprint information of the buildings are derived from Light Detection and Ranging (LIDAR) measurements with a vertical accuracy of $0.5 \mathrm{~m}$ (95\%-confidence limits) [38]. For Paris, we produce a 3-D model of similar thematic and geometric quality. We use building footprint data obtained from the VGI source OpenStreetMap [39]. To obtain building height information we use a normalized digital surface model (nDSM) originating from Cartosat-1 digital elevation models. The approach has been tested against independent ground truth measurements with a mean lateral and vertical accuracy of $6.7 \mathrm{~m}$ and $5.1 \mathrm{~m}$, respectively [40]. From the combination of OSM building footprints and the Cartosat height information, we derive the 3-D city model for Paris at the level of detail 1, i.e., a model that represents buildings by generalized blocks comprising prismatic buildings with flat roof structures. The object-based methodology and the accuracies derived for the 3-D model (with a mean absolute error of $3.2 \mathrm{~m}$ in vertical direction when compared to LIDAR data) are described in detail by [23].

2. Soil sealing information: We use the pan-European soil sealing layer [41] that is derived from high resolution optical remote sensing imagery. This layer measures the continuous degree of soil sealing at a spatial resolution of $20 \mathrm{~m}$, i.e., the percent fraction of areas covered by buildings, streets and other artificial surfaces.

3. Administrative/spatial units: From the European Urban Atlas [42] we use the geometry of building blocks as a reference system. These data are provided for a map scale of 1:10,000 with a minimum mapping unit of 0.25 ha (cp. Section 3.1.2). As administrative boundaries we utilize the accepted standard of Level-2 Local Administrative Units (LAU-2). These entities entail municipalities or the like as a common statistical unit across the 28 EU member states [43]. 
Figure 1 gives an overview of all data sets compiled.
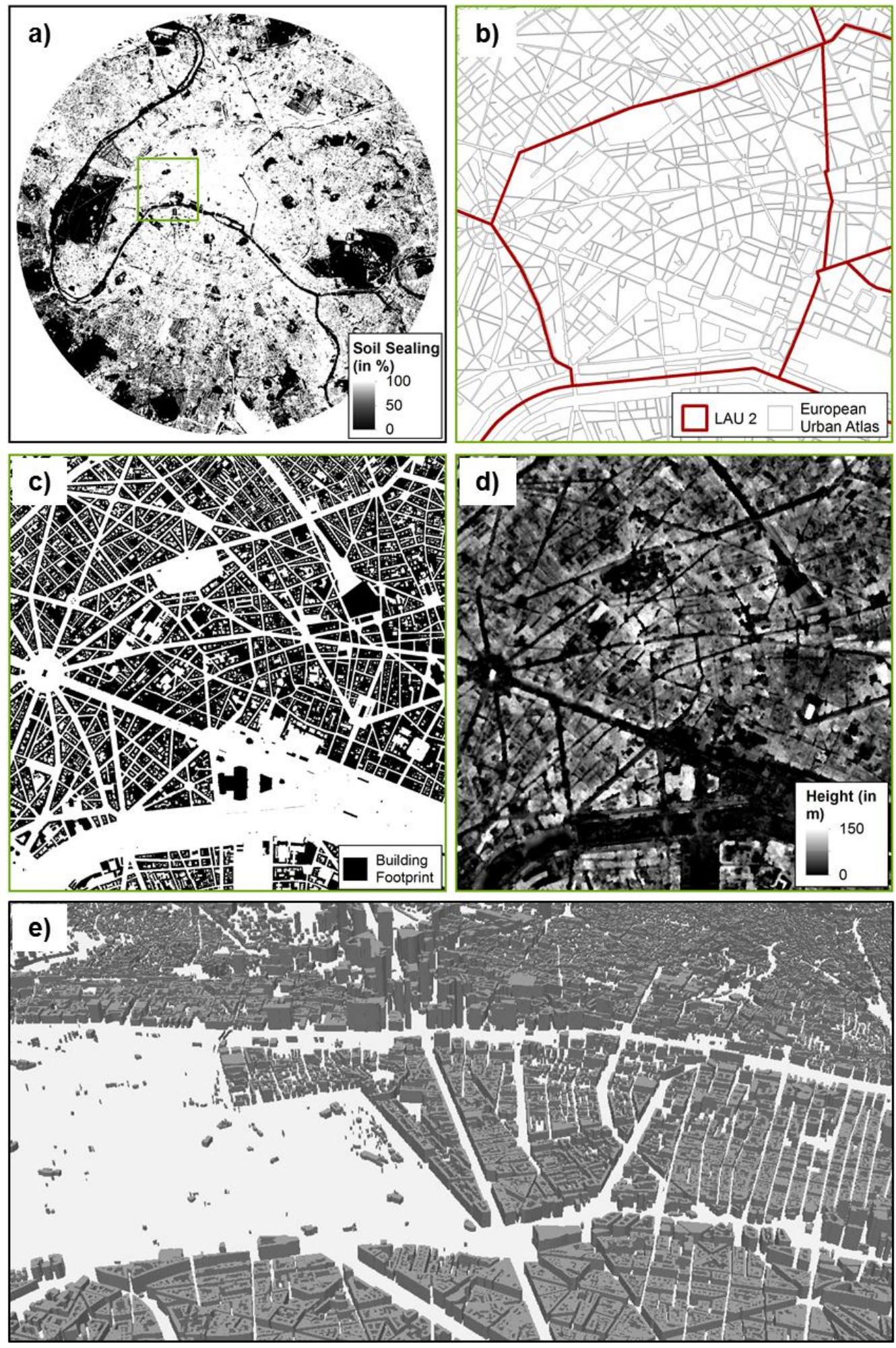

Figure 1. Spatial input data: (a) Soil Sealing Layer; (b) Block units of the European Urban Atlas and Local Administrative Units (LAU); (c) Building footprints from OSM (comparable to the building footprint of the UKMap dataset, respectively); (d) Normalized digital surface model from Cartosat-1 satellite data; and (e) Perspective view of the derived 3-D city model, exemplified for Paris. 


\section{Concepts and Methods}

We introduce a framework for this study to deconstruct physical density of cities (Figure 2). It relates on the one hand to the Concept and Methods Section (Sections 3.1 and 3.2, respectively). In this, various conceptual dimensions of physical urban density, i.e., the thematic (Section 3.1.1), the spatial (Section 3.1.2) and the calculative (Section 3.1.3) dimensions, are addressed and defined. Subsequent analyses of the derived density measures are categorized by means of qualitative or quantitative evaluation methods (Section 3.2). On the other hand, the framework relates to the Results Section (Sections 4.1-4.3). Map appearance (Section 4.1) and the quantitative deconstruction of the density measure are contrasted from non-site-specific (city scale) (Section 4.2.1) and site-specific (zonal model) (Section 4.2.2) perspectives. Ultimately, densities of generic structural types are evaluated by techniques of statistical classification, zonal statistics and by the visual impression from discrete mapping (Section 4.3).

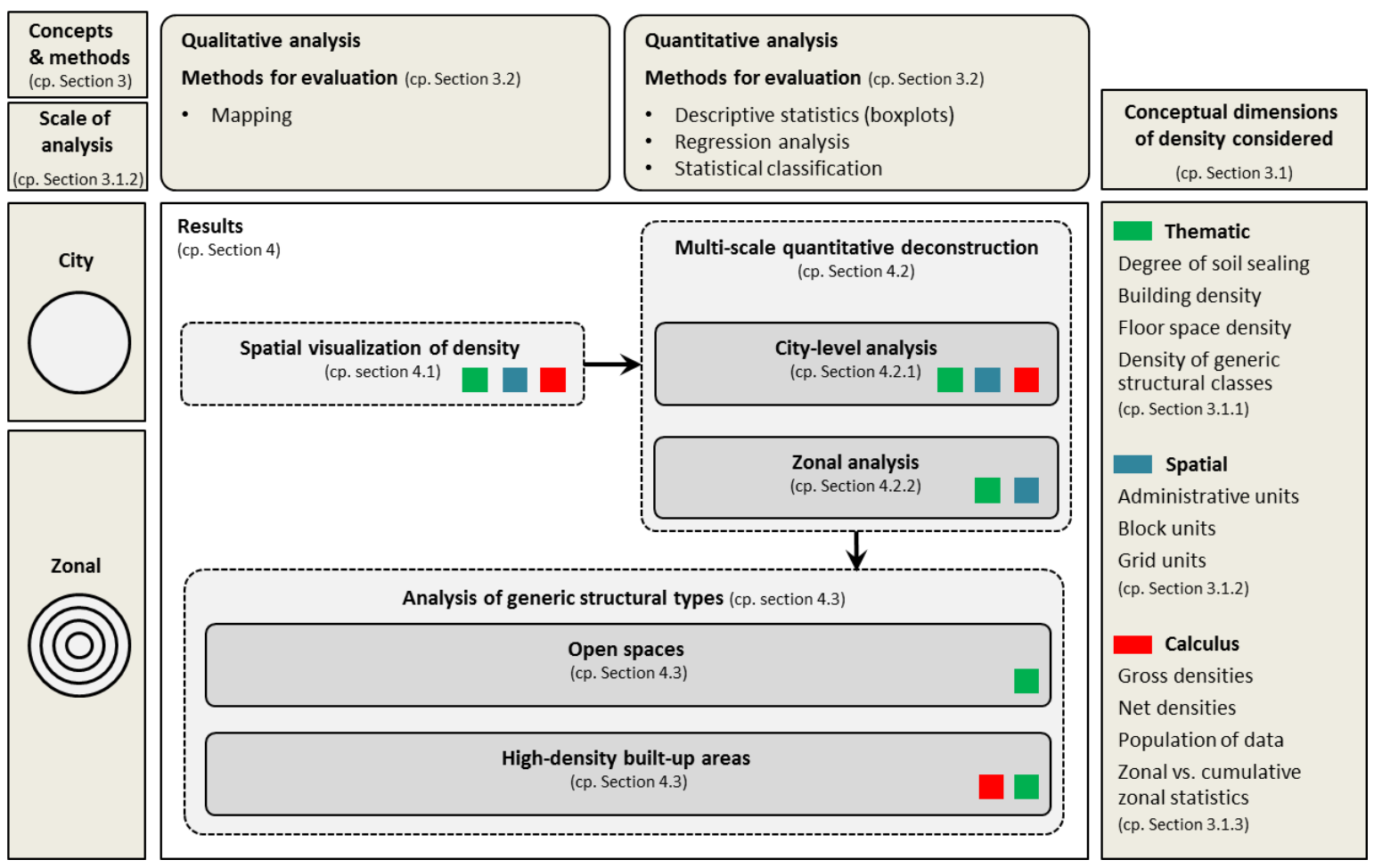

Figure 2. Conceptual framework for a qualitative and quantitative deconstruction of physical urban density.

Due to the multidimensionality of the density measure, an exhaustive presentation of every possible conceptual specification (combination of any thematic, spatial and calculative specifications) is not intended. Nevertheless, we systematically present every possible specification for at least one representative case to evaluate the respective influence of the particular variable combination on its application and interpretation.

\subsection{Conceptual Dimensions of Density}

\subsubsection{The Thematic Dimensions of Density}

From a spatial perspective, density can be calculated for any object with a physical extent. Here, we reduce the various thematic possibilities to characteristics of the built environment. We describe the "physical configuration" by: (1) three different physical density variables; and (2) more specifically, by two generic structural types. 
1. We use two dimensional (soil sealing and building density) and three dimensional (floor space density) variables:

- $\quad$ Building density: This variable corresponds to the cumulative two dimensional extent of all buildings per given reference unit.

- Soil-sealing: This variable reflects the share of impervious surfaces, i.e., the cumulative land cover classes "buildings" and "traffic area" (including parking lots, squares, etc.), per given reference unit.

- Floor space density: This variable derives from the cumulated building ground floor multiplied by the particular number of stories per given reference unit; it is thus a measure for the vertical density of a city.

2. Generic structural types are a certain morphological element within the urban landscape and are thus neither continuous nor area-wide. We exemplify this for discrete classes for two generic structural types: (a) Highest built-up density: we classify this generic structural type using all three variables introduced above. Thus, the respective target class consists of a combination of high density values for each variable. (b) To grasp a concept like density, there is implicitly also the thematic negative, i.e., open spaces, involved [44]. Open spaces do not intrinsically convey the feeling of density but rather the opposite. We explicitly address this perception by including the variable density of open spaces. This measure relates to the spatial share of areas without buildings within the spatial configuration of the city. This parameter thus aims to reflect the publically accessible side of urban space. It shall complement our understanding of spatial density.

\subsubsection{The Spatial Dimensions of Density}

The physical configuration of a city is reflected in a hierarchy of different centers and sub centers or clusters across many scales, from the entire city region to neighborhoods [17]. This complex pattern of diverse, multiscale densities shows that an "objective scale" for the calculation of density is inexistent [4,45]. In this regard the modifiable area unit problem (MAUP) (e.g., [7]) produces a statistical bias by aggregation units that are arbitrary, modifiable, and subjective. We confront this issue by the introduction of a well-defined multiscale spatial concept to deconstruct the sensitivity of analytical results to the definition of the selected reference unit. To do so, we define the spatial dimensions based on the scale of analysis (Section 3.1.2.1) and various reference units (Section 3.1.2.2).

\subsubsection{Scale of Analysis}

The common imagined model of the physical configuration of a city relates to a dense center surrounded by a more or less complex halo of lower-rise buildings and decreasing densities in suburbs. To allow for a differentiated picture of this imagination, we use a multi-scale concept to describe the urban fabric: On the one hand, we use the city scale; we define an 11-km radius circle around a pre-defined center point for the particular city as uniform area of interest. The 11- $\mathrm{km}$ radius is triggered by the consistently available geodata for both cities at the respective extents. We are aware that this spatial unit neither covers the entire metropolitan areas of Paris and London, nor is this unit congruent with the morphologic borders or the respective administrative city limits; nevertheless, this unit allows for a consistent and comparable spatial baseline. Beyond, we believe it captures both, the historic urban cores as well as a significant spatial share of the areas of urban expansion.

On the other hand, we use a more site-specific (zonal) approach for a differentiation of density gradients. Therefore, we apply a ring zone model using one-kilometer rings around the respective center. Due to the definition of our study site based on the spatial data coverage, 10 rings are existent with increasing distance to the center (cp. Figure 3). The center points are pre-defined as the historical centers of both cities: For Paris we use the location of the Notre Dame Cathedral and for London we use the location of St. Paul's Cathedral. 

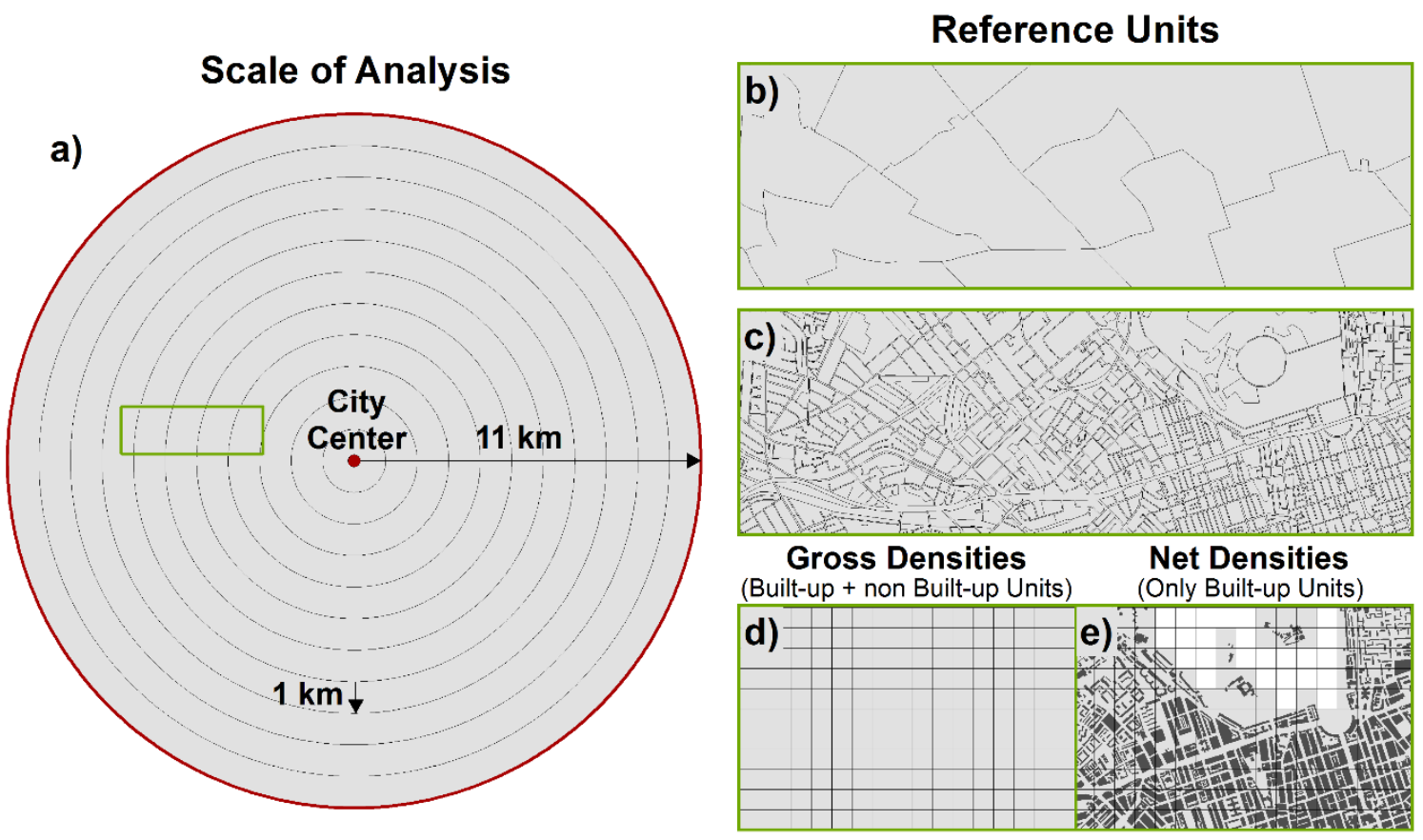

Figure 3. The spatial scales (a); and reference units (b-d) for measuring density for the city scale and the ring model. (b-d) Units of measurement with: (b) administrative units; (c) block units; and (d) grid units. (e) The differentiation between gross and net densities is visualized.

\subsubsection{Reference Units}

The spatial double strategy using the entire study area (11-km buffer) and a site-specific gradient analysis (1-km ring model) is often used for density analysis. However, these two spatial scales do not take the variability of density within the respective reference units into account.

To do so, we calculate density measures using three types of reference units commonly used in the literature: (1) administrative units; these often historically shaped units are man-defined through a political process and are thus often decoupled from the complex physical configuration of the city. Here we use LAU-2 units; (2) block units; in contrast to the administrative units, the block units derived from the European Urban Atlas are small spatial entities delineated through the close meshed street network. While they do not necessarily contain a homogeneous urban morphology, we assume that they are the most appropriate spatial proxy to contain homogeneous physical characteristics within the complex urban environments; and (3) grid units; we use a standard grid geometry. We evaluate the influence of varying grid sizes onto the density measure (cp. Section 3.2). In this regard, the initial standard edge length $(285 \mathrm{~m})$ is derived from the mean size of the block units in the EUA for the test sites.

Figure 3 gives an overview on the two scales of analysis applied in our concept as well as on the definition of spatial reference units. Beyond, differences with regard to the aggregation functions of net and gross densities are visualized which are described in the subsequent Section 3.1.3.

\subsubsection{The Calculation of Density}

Density (D) is the relation between a certain type of class (or object) to the respective reference unit. That is in percent

$$
\mathrm{D}(\mathrm{a})=\frac{\sum a}{A} * 100 \%
$$

where $a$ is a certain thematic class with a spatial extent and $A$ is the spatial extent of the reference unit. Specifically, we calculate three thematic density measures: building density, percentage of soil sealing, and 
floor space density. Beyond, we derive measures of two generic structural types, i.e., highest built-up density and density of open space, as defined in Table 1.

Table 1. Overview of the parameters for measuring physical density.

\begin{tabular}{|c|c|c|}
\hline Physical Parameter & Equation & Variables \\
\hline Building density & $B D_{R}=\frac{\sum_{i=1}^{n_{R}} A_{b_{i}}}{A_{R}}$ & $\begin{array}{c}\mathrm{A}_{\mathrm{b}}=\text { Building Area } \\
\mathrm{R}=\text { Block } / \text { Grid } / \text { Administrative Unit } \\
\mathrm{A}_{\mathrm{R}}=\text { Block } / \text { Grid } / \text { Administrative Area } \\
\mathrm{n}_{\mathrm{R}}=\text { Number of buildings per reference } \\
\text { unit }[\mathrm{i}, \ldots, \mathrm{n}]\end{array}$ \\
\hline Soil Sealing & $S S_{R}=\frac{\sum_{i=1}^{n_{R}} S S_{p_{i}} \times A_{p_{i}}}{A_{R}}$ & $\begin{array}{c}\mathrm{A}_{\mathrm{p}}=\text { Per-pixel area } \\
\mathrm{SS}_{\mathrm{p}}=\text { Per-pixel soil sealing } \\
\mathrm{R}=\text { Block } / \text { Grid } / \text { Administrative Unit } \\
\mathrm{A}_{\mathrm{R}}=\text { Block } / \text { Grid } / \text { Administrative Area } \\
\mathrm{n}_{\mathrm{R}}=\text { Number of pixels per reference unit } \\
{[\mathrm{i}, \ldots, \mathrm{n}]}\end{array}$ \\
\hline Floor space density & $F S D_{R}=\frac{\sum_{i=1}^{n_{R}}\left(F_{b_{i}} \times A_{b_{i}}\right)}{A_{R}}$ & $\begin{array}{c}\mathrm{F}_{\mathrm{b}}=\text { Floor count per building } \\
\mathrm{A}_{\mathrm{b}}=\text { Building Area } \\
\mathrm{R}=\text { Block } / \text { Grid } / \text { Administrative Unit } \\
\mathrm{A}_{\mathrm{R}}=\text { Block } / \text { Grid } / \text { Administrative Area } \\
\mathrm{n}_{\mathrm{R}}=\text { Number of buildings per reference } \\
\text { unit }[\mathrm{i}, \ldots, \mathrm{n}]\end{array}$ \\
\hline $\begin{array}{l}\text { Highest built-up } \\
\text { density }\end{array}$ & $H B D_{R}=\frac{\sum_{i=1}^{n_{R}} A_{H B D_{i}}}{A_{R}}$ & $\begin{array}{c}\mathrm{A}_{\mathrm{HBD}}=\text { Area of highest built-up density } \\
\mathrm{R}=\text { Block Unit } \\
\mathrm{A}_{\mathrm{R}}=\text { Entire } / \text { Ring Area } \\
\mathrm{n}_{\mathrm{R}}=\text { Number of blocks of highest } \\
\text { built-up density }[\mathrm{i}, \ldots, \mathrm{n}]\end{array}$ \\
\hline Density of open spaces & $D O S_{R}=\frac{\sum_{i=1}^{n_{R}} A_{O S_{i}}}{A_{R}}$ & $\begin{array}{c}\mathrm{A}_{\mathrm{OS}}=\text { Area of an open space } \\
\mathrm{R}=\text { Block Unit } \\
\mathrm{A}_{\mathrm{R}}=\text { Entire } / \text { Ring Area } \\
\mathrm{n}_{\mathrm{R}}=\text { Number of blocks classified as open } \\
\text { space }[\mathrm{i}, \ldots, \mathrm{n}]\end{array}$ \\
\hline
\end{tabular}

The calculation of density seems mathematically straightforward; however, the following three specifications shall explain special scenarios:

1. We differentiate between net and gross density; net density refers to densities where the reference units applied exclude certain areas. In our case, we calculate net density only using all reference units which contain "buildings". Thus, administrative units, blocks or grids not featuring the respective object "buildings" are excluded from the calculation and the subsequent analysis. In comparison, gross densities operate on the total number of reference units [5]. Figure 3d,e (for gross density and net density, respectively) visualize this differentiation.

2. The density measures may vary significantly with respect to the population of data used. As example, when referring to generic structural types in cross-city comparisons their spatial classification differs depending whether one refers to the global population of data (e.g., across all considered cities) or if their classification relates to only one population (e.g., per city) at a time. The resulting density measures may reveal significant differences.

3. For the zonal model we test two different strategies: The calculation of density measures per individual ring and for a cumulative reference; the latter means we calculate density measures for the reference unit of the first ring, then for the first and second rings combined, and so on. 


\subsection{The Evaluation of Density}

The manifold parameters possibly combined to derive the measure density-may it be thematically, spatially, or calculative-make its application complex. We introduce an evaluation procedure that combines mapping, quantitative, thematic, spatial and intrinsically geographic approaches.

We qualitatively examine city configurations by mapping the calculated density measures for a visual comparison and interpretation. In doing so, we also contrast the influence of various reference units. Geographically we compare the spatial configurations of cities with regard to different considered physical density variables. With it we aim at demonstrating the fuzziness of density maps and their related meaning. Additionally, we visualize examples of typical generic structural types in ground figure plans.

We employ several statistical techniques for a quantitative deconstruction of urban density for both the city and zonal scale:

First, we calculate the distribution of density values and visualize them as boxplots. The boxplots are defined by the standard statistical measures median, interquartile range and standard deviation. With this, we aim to evaluate absolute and relative influences of possible conceptual specifications (combination of thematic variables, scales of analysis, reference units, and calculation methods) onto the density measure.

Second, we extend the quantitative analysis by the examination of correlations between thematic variables. In this, we empirically determine the coefficient of correlation for the series of measurements at the various spatial units [46]. The robustness of correlation between independent and dependent variables can be evaluated by means of the coefficient of determination $R^{2}$. The measure describes the explained variance of a dependent variable utilizing a specific regression model [47]. In our case, the coefficient of determination $\left(R^{2}\right)$ is described by a linear regression model of first order [46].

$$
\begin{aligned}
r^{2} & =1-\frac{R S S}{T S S} \\
\mathrm{RSS} & =\sum_{i=1}^{n}\left(d_{i}-\hat{d}\right)^{2} \\
\mathrm{TSS} & =\sum_{i=1}^{n}\left(d_{i}-\bar{d}\right)^{2}
\end{aligned}
$$

where RSS and TSS are the residual and total sums of squared errors between two density measures, respectively; $\mathrm{n}$ is the number of observations; $d_{i}$ is an observed value; $\bar{d}$ is the mean of all observations; and $\hat{d}$ is the response of the regression model. $\mathrm{R}^{2}$ equals 1 if all points in the scatterplot show a perfect linear relationship between the two considered data sets. We visualize the scatterplots of these bivariate distributions. This allows for an evaluation of the deviations of the thematic dimensions regarding density.

Third, we systematically analyze the effects of reference units (the MAUP problem) onto the density measurements. Therefore, we calculate on the one hand the variability of the reference units for the London and Paris test cases in terms of their spatial extents. The variabilities of these aerial measures are presented as boxplots. With it, we aim at an interpretation of how meaningful those units may be. On the other hand, we systematically analyze the influence of the applied grid sizes on the calculated density measures. We use the grid width of $285 \mathrm{~m}$ as a standard within the analysis. However, to analyze the influence of varying grid sizes, we additionally calculate smaller $(35 \mathrm{~m}, 85 \mathrm{~m}$, $135 \mathrm{~m}, 185 \mathrm{~m}, 235 \mathrm{~m})$ as well as larger grid widths $(500 \mathrm{~m}, 750 \mathrm{~m}, 1000 \mathrm{~m}$, and $1500 \mathrm{~m})$ to reveal the effects on the density measure.

Ultimately, we analyze density measures for generic structural classes, which are in contrast to the other variables not area-wide and continuous. This may add to the understanding of the density 
measure, as e.g., a city may have on average a higher building density than another; however, it is not an obligatory consequence that the share of open spaces is in turn lower. The generic structural classes are a spatial selection of areas from the global population. Thus, we compare here only spatial shares of the entire city configuration. Our analysis here is two-fold: For the class highest built-up density, we identify areas with values higher than the 85th quantile for all the three continuous variables-building density, soil sealing and floor space density. However, to account for the variability of the density measure with respect to the population of data used, we relate the analysis to both the global population of variables across cities, and the per-city populations. For the analysis we use zonal statistics to evaluate results based on the two different calculation methods. Regarding the class open spaces, we define that an open space has a building density lower than $1 \%$. We furthermore specify whether the open space is a natural open space (e.g., a park) or a sealed surface by using a threshold based on the soil sealing layer. In particular, we consider any open space identified as natural if the share of soil sealing is lower than $10 \%$. We are aware that those thresholds may be subjective. However, as we apply them in a consistent manner for both cities, we argue that this is appropriate for a cross-city comparison. The analysis on open spaces then uses zonal statistics to evaluate the density measure.

\section{Results}

In this section, we deconstruct the density measure to evaluate its fragility in dependence of selected dimensions and methods of calculation.

\subsection{The Mapping of Density}

The cartographic visualization of density is a powerful tool in urban planning. However, when mapping densities, it becomes obvious that map appearances vary significantly with respect to the thematic dimension, the scale of analysis, the applied reference unit or the method of calculation. Figure 4 illustrates the variability of the density measures regarding building density, soil sealing and floor space density for the entire areas of investigation in Paris and London at block level.

In general, both cities show maximum building densities in their respective geographic centers. However, the cities also reveal differences in density patterns: The extent of highest building densities is significantly larger for Paris. Within the Boulevard Périphérique-an administrative (and obviously also a morphological) demarcation line in Paris' city configuration-densities are continuously very high. Outside this area, building density decreases as function of the distance to the city center. For London the spatial extent of high building densities are significantly smaller and basically limited to the central areas north of river Thames, between the so-called "City" (east) and Hyde Park (west).

Soil sealing densities show a partly different spatial pattern. Similar to building densities, high soil sealing densities are mapped for the urban centers. However, the decrease in soil sealing with rising distance to the urban center is by far less distinct. In Paris the decrease of soil sealing even outside the Boulevard Périphérique is marginal. For London, areas of high soil sealing comprise a significantly larger area than high building densities; however, the decrease to the peripheral areas is apparent.

The floor space density reveals a different, more complex spatial pattern: While a generally decreasing floor space density from the center to peripheral areas is also given, this gradient is significantly interrupted by local maxima of urban mass concentration. As examples, the La Defense area northwest of the city center in Paris (a planned business district with an agglomeration of high rise buildings) clearly reveals an urban mass concentration outside the Boulevard Périphérique. For London, peripheral business districts such as Canary Wharf east of the City reveal the existence of morphological sub-centers. 


\section{Paris - Building Density}

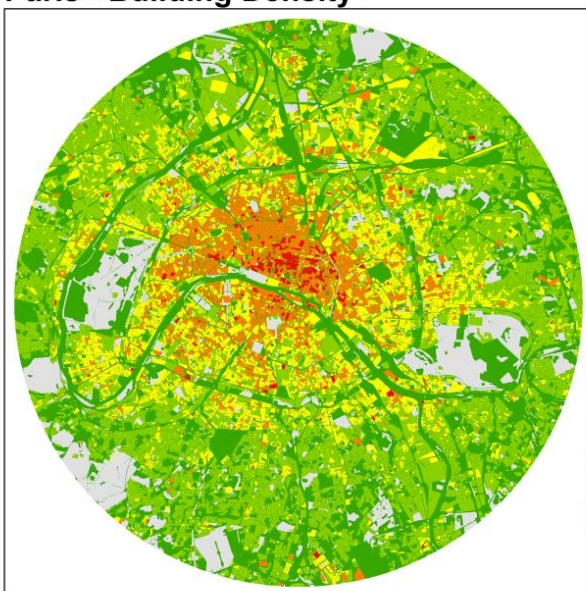

Paris - Degree of Soil Sealing

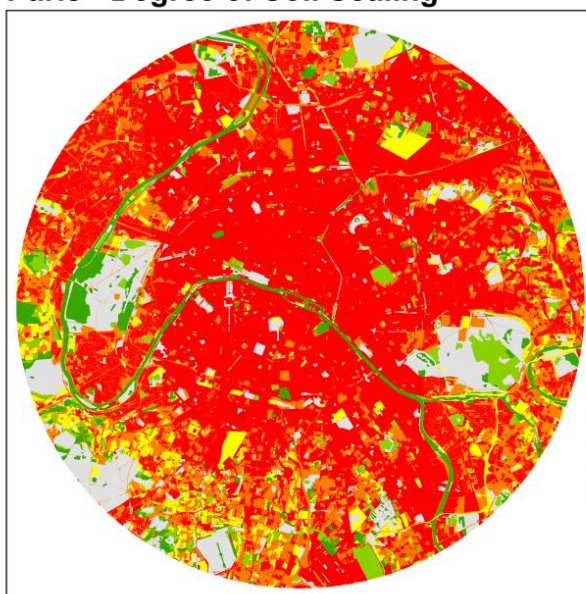

Paris - Floor Space Density

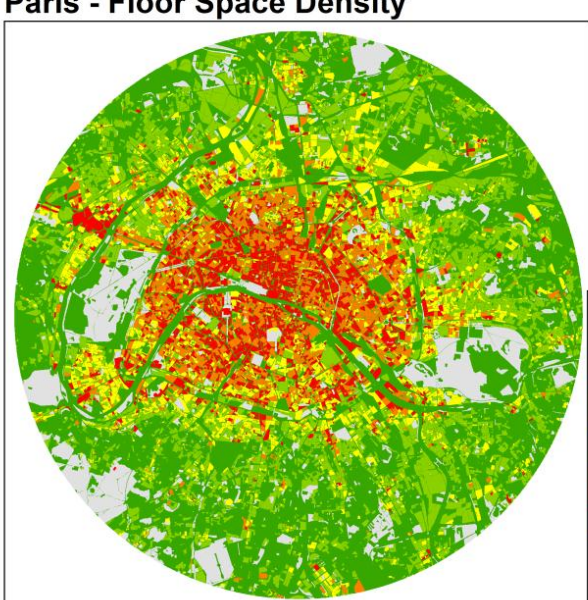

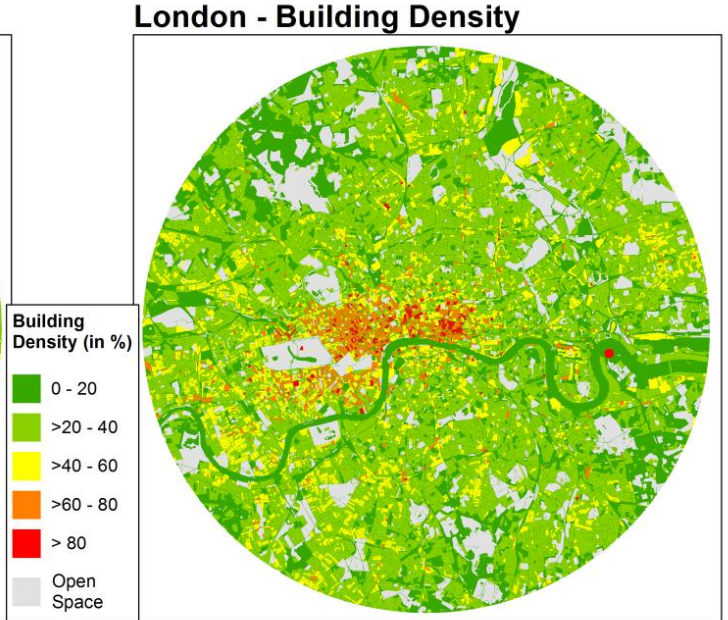

London - Degree of Soil Sealing

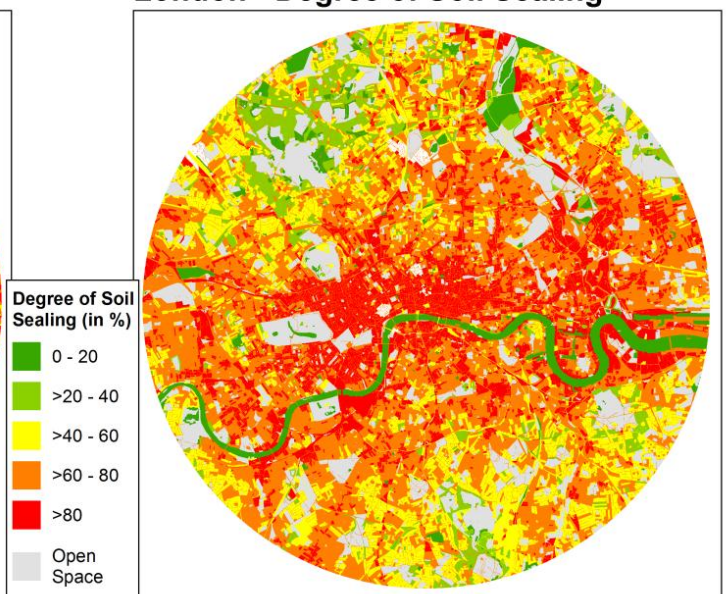

London - Floor Space Density

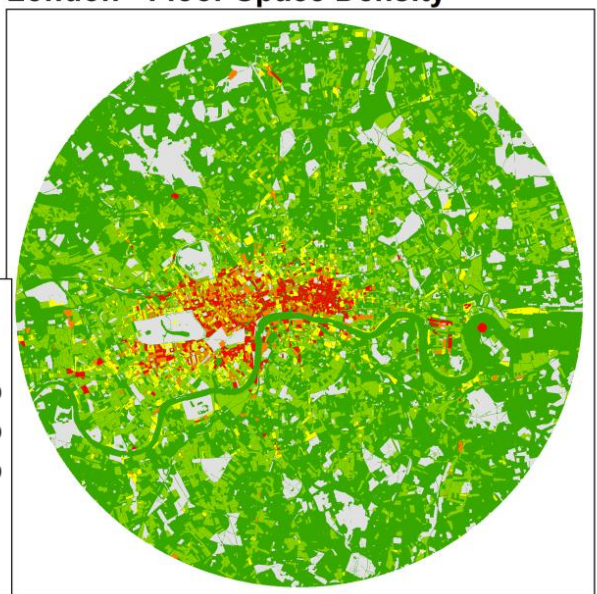

Figure 4. Density maps at block level for Paris and London: (a) Building density; (b) Soil Sealing; and (c) Floor Space Density.

The mapping of three different spatial variables underpins the variability of density with respect to the particular thematic measure. This is even true, if parameters relate to the same physical ground objects such as buildings as it is the case in terms of building and floor space density. While Figure 4 presents the map appearances of thematic density measures on block level, Figure 5 illustrates the variability of density measures with regard to the choice of particular reference units. This is 
exemplified by the thematic parameter building density mapped on the level of administrative and grid units for Paris.

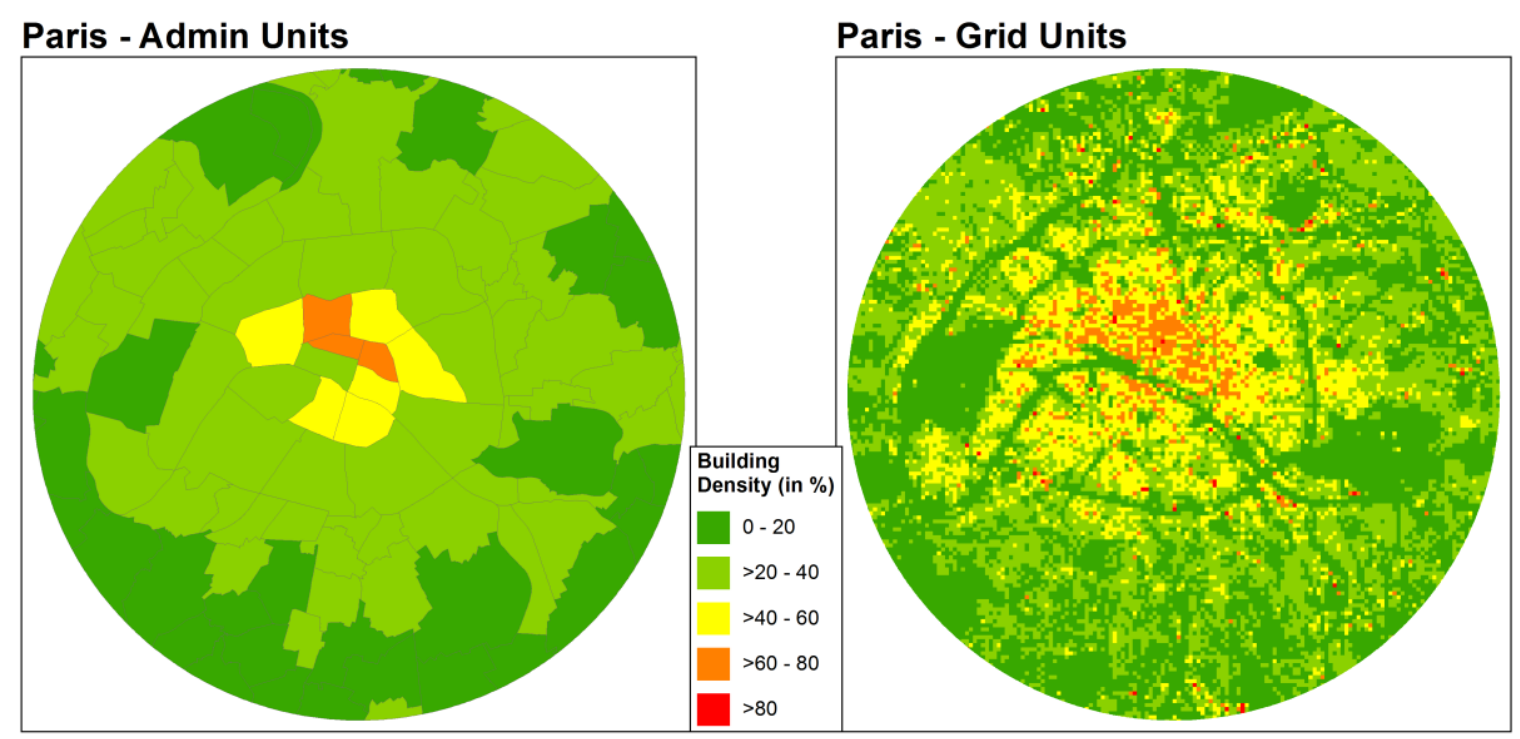

Figure 5. Building density maps related to different reference units: grid vs. administrative units.

The comparison of map appearances for the parameter building density in dependence of the reference units (grid level vs. administrative unit (Figure 5) vs. block level (presented in Figure 4)) illustrates their significant influence. Due to the chosen grid dimension, which is related to the average size of block units, both reference units reveal a similar spatial density pattern. One might conclude that both block level and the used grid level capture the change of complex, small-scale urban morphology. In contrast, administrative units blur the real physical configuration of the city. While the general decrease in building density from the city center to peripheral areas is preserved, the true urban morphology configuration is hidden due to the large size of spatial units and their inappropriateness to represent the true urban morphology. Thus, the selection of an appropriate scale of measurement is crucial.

\subsection{Quantitative Deconstruction of the Density Measure}

\subsubsection{The City Scale}

We start the quantitative deconstruction of density using the three variables building density, soil sealing and floor space density for three reference units (administrative, grid and block units) as well as for two calculation methods (gross and net density). Figure 6 illustrates these results as boxplots at city level.

Figure 6 allows the following conclusions: (1) Naturally, net densities are consistently higher than gross densities. Consequently, the application of either of the measures has influence on the absolute values of density measured. The differences vary between $0 \%$ and $4 \%$. Thus, whenever used for cross-city comparisons the specification of the measurement of density is imperative. (2) Per definition, soil sealing density values are significantly higher than building density values. It is interesting to note, that for the specific cases of Paris and London, the cross-city differences in soil sealing values are significantly higher (in the range of $20 \%$ depending on the reference unit) than for building density (in the range of $4 \%$ ). (3) The administrative units result in the comparably lowest density values. In contrast, the block units reveal the highest values. The assumption suggests that this is due to the larger sizes of administrative units. However, as Figure 7 contrasts the dimensions and variabilities of block and administrative units, this assumption is only partly true. Indeed, the administrative units 
for Paris are significantly larger than the block and the used standard grid units. For London, the opposite case of smaller LAU-2 units is given. Although the spatial dimension of grid and block units is similar, their difference in spatial form reduces the density values for grid units by a range of $10 \%$. This can be explained by the spatially unspecific form and location of grids neglecting the real urban physical configuration (which is supposed to be better in block units). (4) Geographically the results clearly reveal that at city level Paris is denser than London, both in terms of the observed medians and the peak values. This holds true for the thematic dimension of the parameters building density, soil sealing as well as the floor space density. It holds true for both net and gross densities across all three reference units applied. Furthermore, the variance of density values is commonly higher in Paris-with soil sealing densities as the only exception.

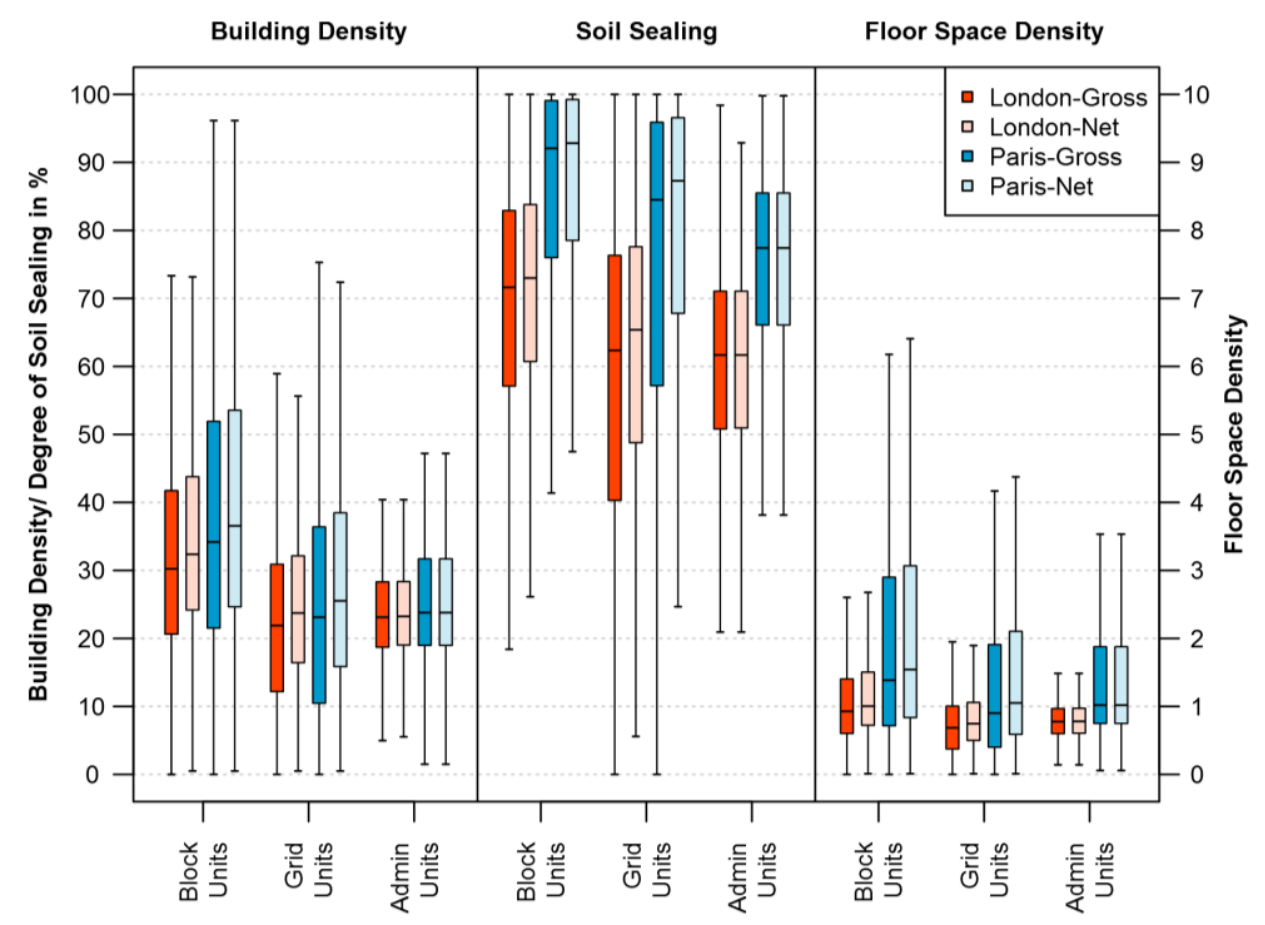

Figure 6. Density measures illustrated as boxplots for three thematic dimensions-building density, soil sealing, and floor space density-and the influence of the reference units-administrative, grid and block units—calculated for gross and net densities at city scale for Paris and London.

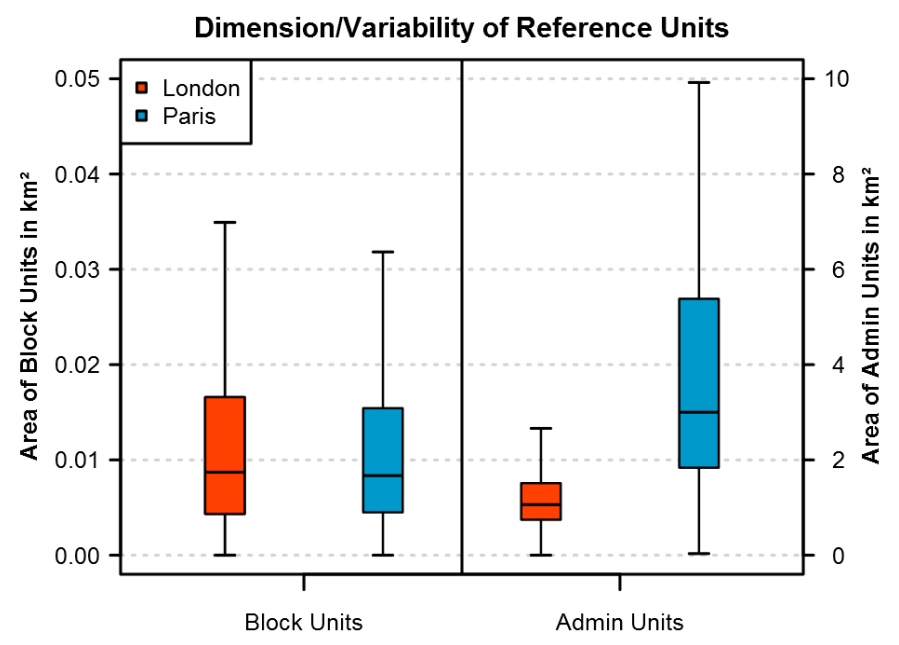

Figure 7. The influence of varying grid sizes on density measures calculated for gross densities. 
In addition to the explanation above, Figure 7 reveals the high spatial variability of administrative units across cities. This fact emphasizes the conclusion that administrative units are not suitable entities for density measures and their comparison across cities.

From the previous results, it is already obvious that no linear correlation between the three spatially continuous thematic variables applied in our study exists. Figure 8 plots pairwise the respective thematic dimensions against each other at block level.

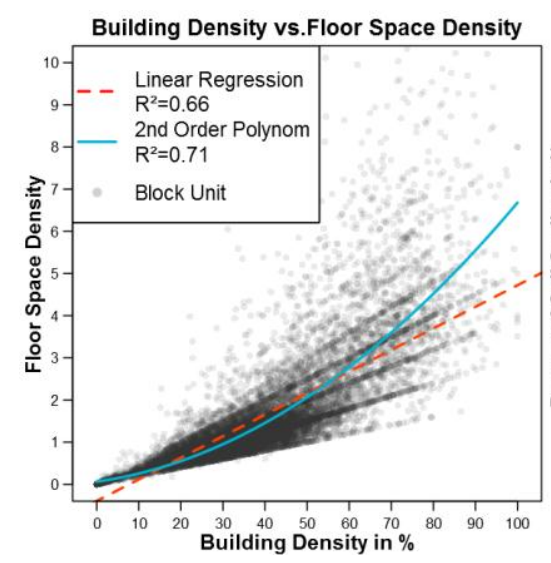

(a)

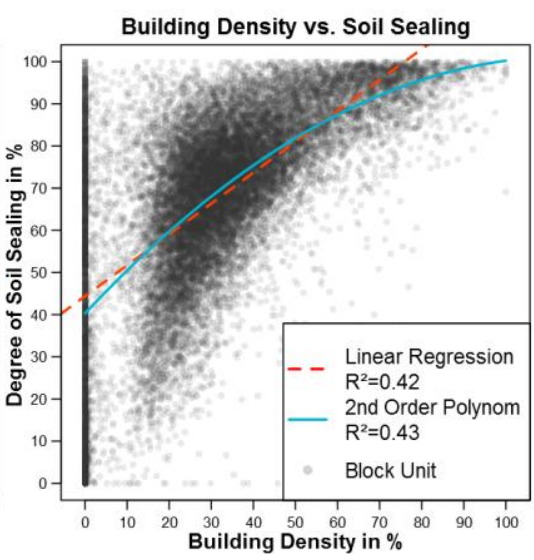

(b)

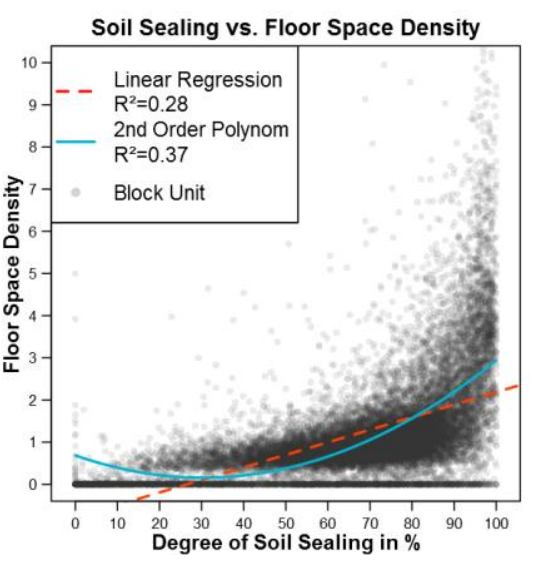

(c)

Figure 8. (a) Floor space density as function of building density; (b) Soil sealing as function of building density; and (c) Floor space density as function of soil sealing; all examples are presented at block level using gross densities for Paris.

Although all applied variables belong to one family describing physical characteristics of the urban landscape, no linear correlation is evident. However, the coefficients of correlation show positive relationships. Especially the two variables that describe the building stock-building density and floor space density-show a high correlation with an $\mathrm{r}^{2}$ of 0.66 in linear regression. However, this does not mean knowing either of the two variables is a reliable predictor for the other. Regarding soil sealing and building density a medium correlation $\left(\mathrm{r}^{2}=0.42\right)$ and for soil sealing and floor space density a low correlation $\left(r^{2}=0.28\right)$ is calculated from linear regression. This quantitatively confirms criticism on the EUA for hiding information on the true urban morphology (e.g., [48]), as its density classes are based solely on soil sealing. Thus, conclusions on the urban morphology based on one variable may be misleading.

All previous analyses in our study have been done on a fixed standard grid size, or on the invariable sizes of blocks or administrative units. Addressing the MAUP problem, we aim at evaluating the effects of varying grid sizes on density measures. Therefore we systematically quantify the variation of density values with alternating grid sizes. Figure 9 illustrates the variation of density values at city level for three thematic dimensions in dependence of varying grid sizes.

The analysis reveals a natural trend of decreasing density variances across thematic dimensions with increasing grid sizes. Rising grid sizes obviously are more likely to contain a mixture of structural types leveling local variations of density throughout urban morphology. At the specific thematic levels, a clear trend is only given for the variable soil sealing: with increasing grid sizes, median density values decrease. This trend is only marginal for the other two thematic layers. For building density and the floor space density, median values are unpredictable with respect to the applied grid sizes. 


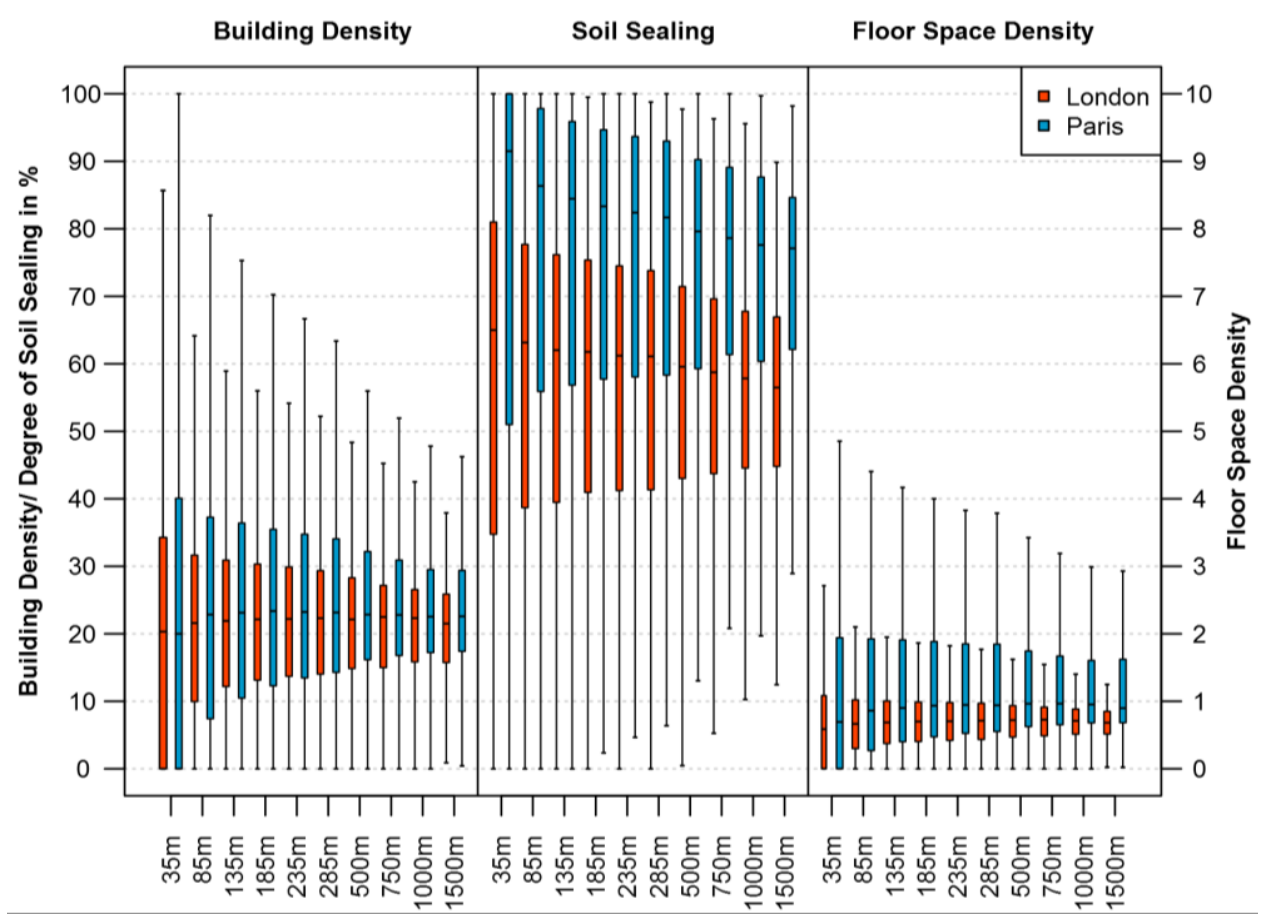

Figure 9. The influence of varying grid sizes on density measures calculated for gross densities for London and Paris.

\subsubsection{The Zonal Scale}

The high variance of density values for the entire study areas implies an uneven distribution of physical density in the city configuration. Figure 10 illustrates the site-specific gradient analysis that reflects this uneven spatial distribution more clearly. While the zonal statistics calculated for individual rings are presented for the three thematic dimensions at block level, for one example-building density-the cumulative gradient analysis is carried out to evaluate its influence on the density measure. Furthermore, to account for the variance of density measures due to the chosen reference units, two additional examples are presented: for the thematic variable soil sealing at the level of administrative units and for the thematic variable building density at grid level.

The basic imagination of the physical configuration of a city-high dense center and decreasing densities with rising distances-is generally confirmed. Based on the median, we find with rising distances to the centers predominantly a constant decrease of gross densities values regardless of the thematic dimension or the calculus (ring vs. cumulative gradient analysis). While this is generally true for block as well as grid units, it is interesting to note that the administrative units reveal high irregularities to this trend; it is an additional proof for the spatial units to be inappropriate for measuring density - which goes along with the blurring effect in map appearance. Furthermore, it is interesting to note that for the cumulative gradient analysis, the decrease of density is not as distinct, and naturally the absolute density values are higher. This is of course founded in the alternative reference units constantly integrating the center; however, with more than $10 \%$ higher values in the outer areas, this calculation method reveals the fragility of the measure and its interpretation.

Geographically, the result again reveals that at all thematic and spatial dimensions Paris is denser than London. On a closer look, significant differences in density configurations between Paris and London can be identified as well. While the urban center in London reveals the highest median densities measured for building density as well as for floor space density, in Paris the highest median densities are not found in the center but in the 1-2 km distance. Beyond this, the highest variance and peak values are identified for the inner 2-3 rings (depending on the variable) with a decrease in both, variance and peak, with rising distance. For Paris, neither the highest variance nor the highest 
peak density values are found in the inner three rings. This implies a more heterogeneous density structure for Paris in the outskirts and vice versa for London in the downtown area. In Paris, the highest variances are identified in a distance from 3-6 km distance for both, building density and floor space density. For example, in a distance of $4-5 \mathrm{~km}$, the median building density in Paris is with $56 \%$ compared to $32 \%$ in London significantly higher. Last but not least, the density in Paris is significantly higher within the first 5-6 rings compared to London, while in the outer rings the densities assimilate across cities.
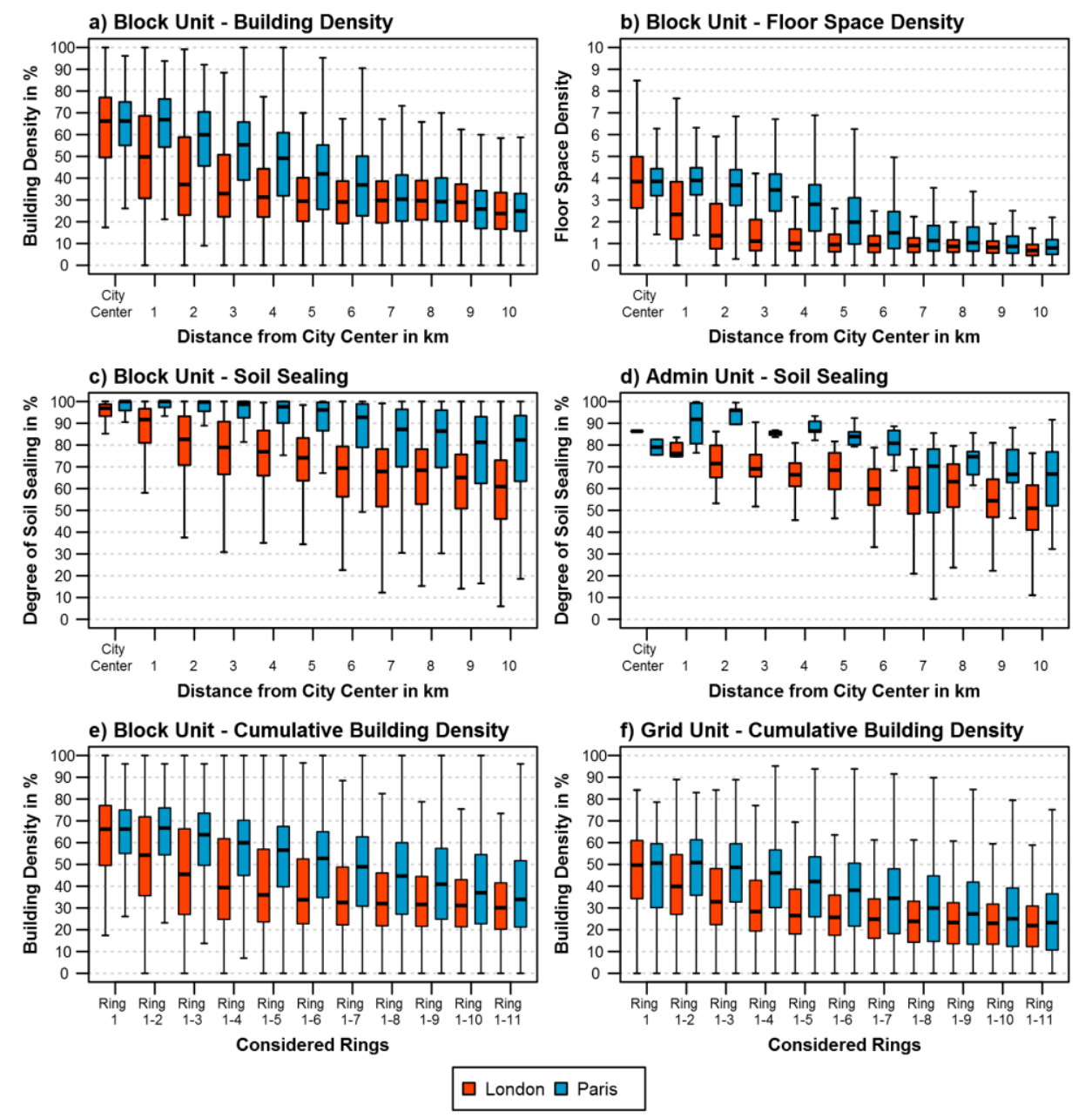

Figure 10. Gradient analysis for three thematic dimensions illustrated by site-specific boxplots on: (a) building density; (b) floor space density; and (c) soil sealing at block level for London and Paris. (d) For one example, soil sealing, the gradient analysis is presented at administrative units. For two other examples, using building density, the cumulative gradient analysis is illustrated for: (e) block units; and (f) grid units.

\subsection{The Density of Generic Structural Classes}

To this point of the study, all results on density are related to an area-wide, continuous understanding of the city configuration. However, as introduced in Section 3.2, the measure of density can also be applied to specific parts of the city. In our case, we exemplify this by the analysis of two generic structural classes, which naturally exhibit a limited area of the entire city: highest built-up densities and open spaces.

Figure 11 illustrates the map appearances of the two classified generic structural classes at block level. Furthermore, the gradient analysis of the two classes is visualized. For the class highest built-up density the differences with respect to the population of data used (globally, i.e., across cities, and per 
city) are plotted. For the class open spaces an additional specific sub-classification in sealed open spaces and green open spaces is calculated and plotted.

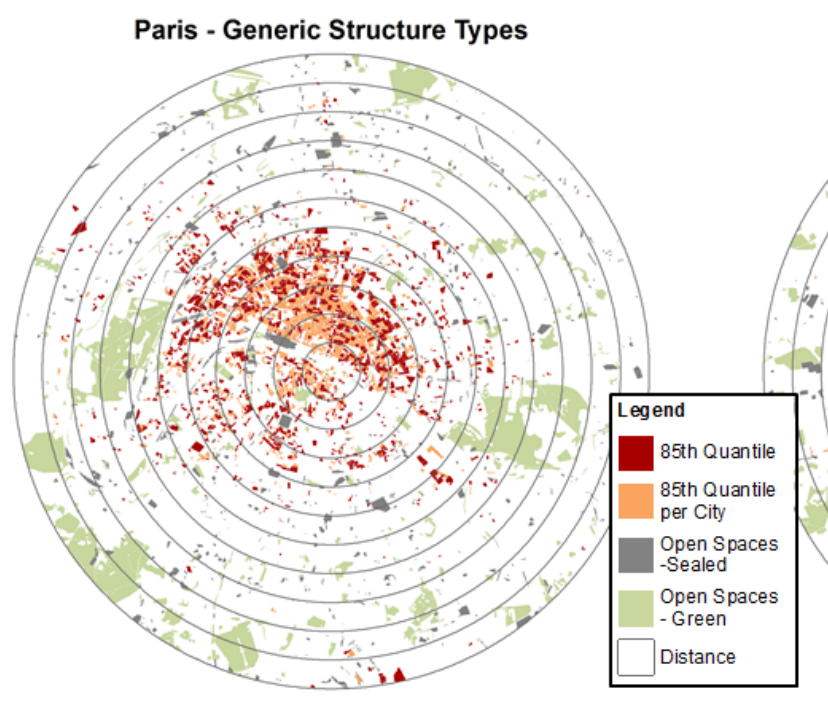

Density of Generic Structure Types - Paris -

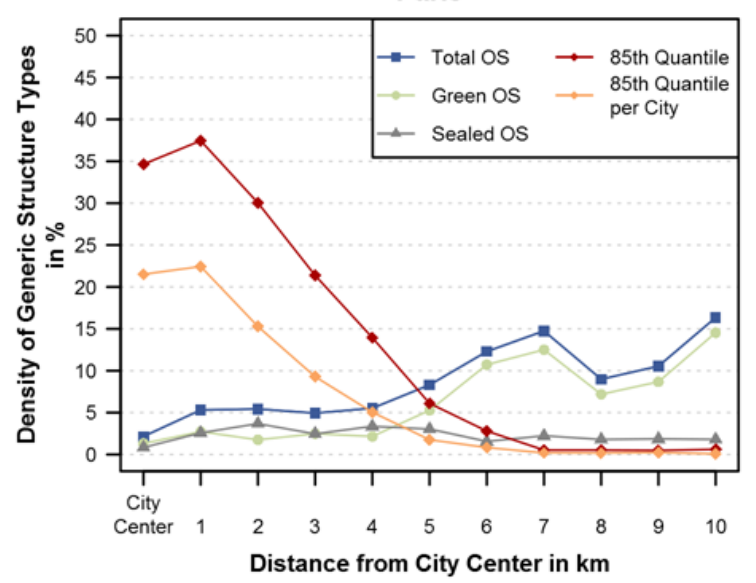

London - Generic Structure Types

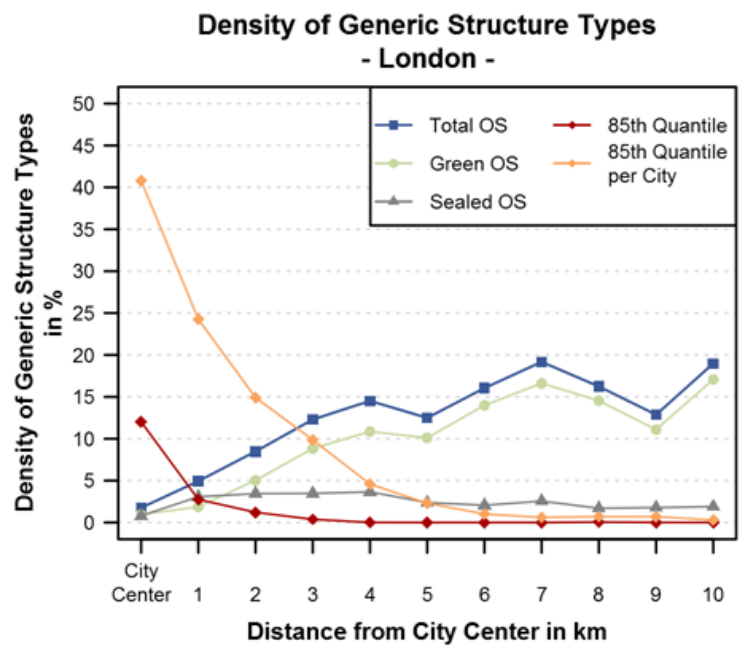

Figure 11. Mapping appearance of generic structural classes (open spaces and highest built-up density) at block units; gradient analysis of the two generic structural classes (specified for open spaces in sealed and green open spaces).

The following conclusions can be drawn from Figure 11: Regarding the generic structural class highest built-up densities, the applied statistical approach drawing from the global population of data for both cities allows for a direct approach comparing cities. While the analyses above reveal that built-up density of Paris is significantly higher, this type of analysis emphasizes this result. The share of these areas is in Paris with 5.91\% of the entire study area significantly higher than for London, where it amounts only $0.25 \%$. In Paris, this specific generic structural type is dominant within the Boulevard Périphérique and covers a large share of the inner city. In London, this class is only slightly represented in the central parts north of river Thames. In absolute numbers the difference in density between both cities based on this specific structural type becomes even clearer: with $22.5 \mathrm{~km}^{2}$ we identify 23 times as much in extent for Paris ( 2670 blocks) than for London $\left(0.95 \mathrm{~km}^{2} ; 221\right.$ blocks). Regarding zonal statistics, it becomes obvious that the urban mass concentration in the center of Paris is significantly higher and covers larger spatial extents than in London. When changing the applied statistical approach by drawing from the population of data for one city at a time, the results are reversed. The share of areas in London is then with $3.12 \%$ of the area of interest higher than for Paris 
(2.63\%). With $11.86 \mathrm{~km}^{2}$, it is larger than that of Paris $\left(9.98 \mathrm{~km}^{2}\right)$. The analysis reveals that London also has-compared to the surrounding urban morphology—a distinct urban mass concentration in the city center. This particular result reveals the methodological and analytical fuzziness of the density measure. It shows that varying calculation methods may lead to contradictory results. It shows that the spatio-structural manifestation of density always needs to be seen in a relative manner.

With regard to open spaces, our natural perception of this structural class does not correspond to density. Nevertheless, the spatial share of open spaces is also an expression of density. Here, open space density is higher in London, with a share of $15.09 \%$ within the area of interest compared to $10.73 \%$ for Paris. The difference mainly relates to the share of green open spaces: While the share of open spaces with impervious surfaces is more or less equal across cities $(2.20 \%$ for Paris; $2.30 \%$ for London), the share of green open spaces is significantly higher in London $\left(8.53 \%=32.43 \mathrm{~km}^{2}\right.$ for Paris; $12.79 \%=48.62 \mathrm{~km}^{2}$ for London). In zonal statistics, the share of total open spaces generally increases with rising distances to the city center, revealing its inverse spatial character with regard to the analyzed measures on the built-up. While this increasing trend is generally true for the share of green open spaces, the share of sealed open spaces contains about the same small share (around $1 \%-2 \%$ ), more or less independent from the location. This effect is evident for both cities. On a closer look, the share of sealed open spaces from the total share of open spaces is only higher in the central areas (first four rings for Paris and first two rings for London); with rising distance to the city center natural open spaces are dominating (e.g., $17.5 \%$ in the peripheral ring in London for natural open spaces vs. $1 \%$ for impervious surface open spaces).

The deconstruction of density has been conducted on two scales of analysis. While the original data sources are 3-D building models, density has been analyzed on aggregated units. In a final analysis, we aim to capture the original urban morphology at building level to visually illustrate density not in an abstract manner but in the real building configuration. Therefore we visualize typical generic structural types in ground figure plans as well as 3D city models, taking examples from both cities. With it, we aim to qualitatively illustrate the building pattern and their intrinsic density-as a representation of physical reality. Figure 12 exemplifies three generic structural types per city: highest building density, average building density and low building density and gives perspective views on these three structural types using the 3-D city models.

In Paris, the highest building densities are found in the quartiers near the city center in the 8th and 9th arrondissements. Closed block structures consisting of 6-7 story town houses with additional buildings in the respective inner courtyards mainly show building densities higher than $70 \%$. These block developments are ordered between the structuring boulevards laid out by Haussmann's reorganization of the city morphology (Figure 12a). Medium building densities of row and detached houses in Villetaneuse, Paris (Figure 12b). Low building density north of the city center. The area shows a morphological transition from $4-5$ story buildings made with precast concrete slabs in winding formations of a planned banlieue in Saint Denis, Paris (west) to two story row houses with private gardens (east) (Figure 12c). Wilhelminian style quarter between London Soho and Mayfair. The high building density in the city center of London is defined by large buildings in traditional block perimeter development (Figure 12d). Typical row-house settlement in Battersea Borough, London. The compact development with terraced two to three-story row houses leads to medium building densities in that area (Figure 12e). Low density residential area in Tottenham, London. The unique typology of two-story townhouses that contain private gardens is a characteristic London housing style resulting in low density urban morphology (Figure 12f). 3D city models for: an agglomeration of high rise office buildings at business district La Defense, Paris (Figure 12g); medium dense housing development with detached, semi-detached and apartment buildings in Vésinet, Paris (Figure 12h); and low density settlement with scattered row house structures including medium sized private gardens in Purley, London (Figure 12i). 

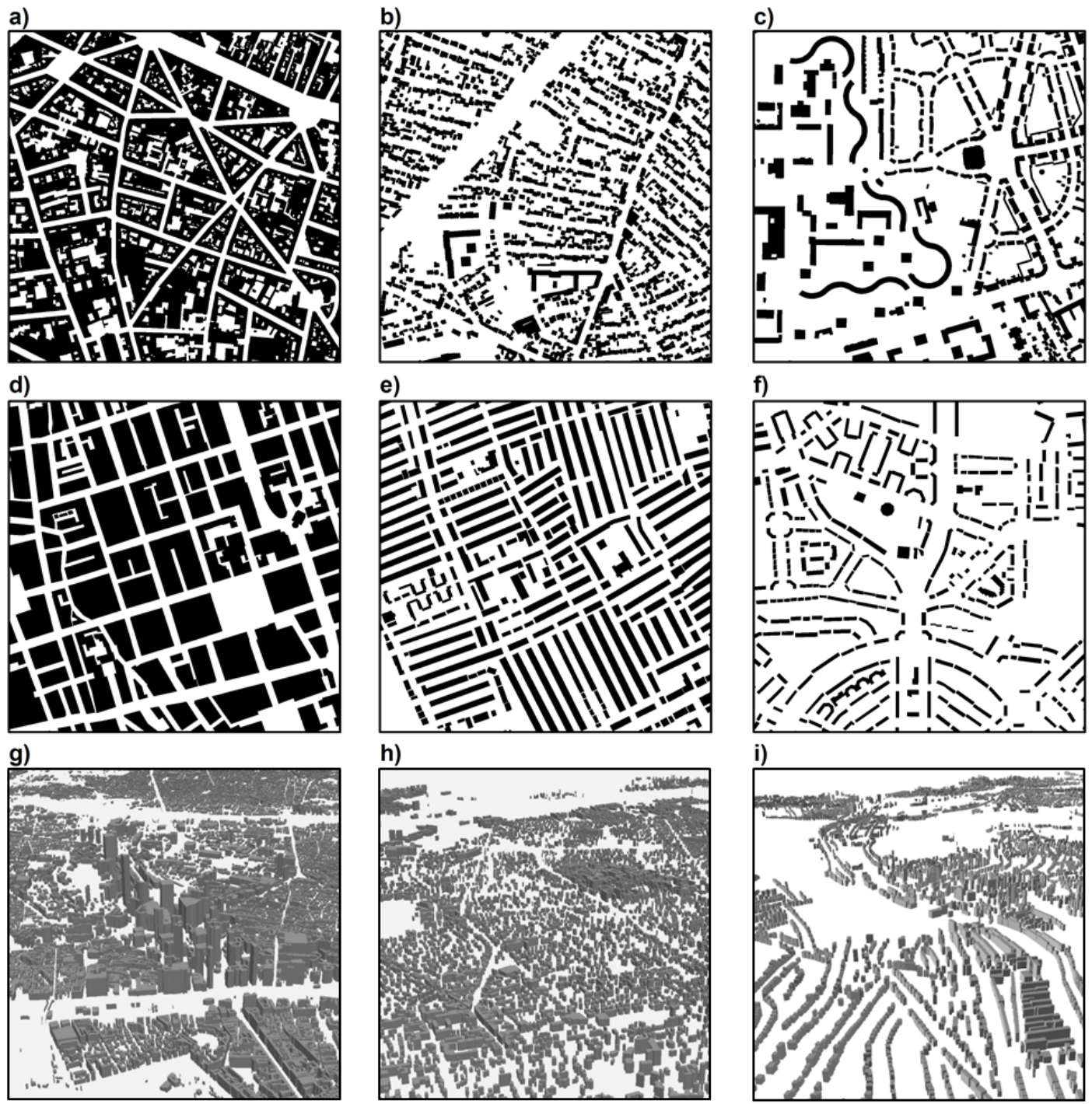

Figure 12. Ground figure plans for characteristic generic structural types: High building density for (a) Paris and (d) London; Medium building density for (b) Paris and (e) London; Low building density for (c) Paris and (f) London; (g) High floor space density and (h) medium density housing development in Paris; and (i) row house structures in suburbs (Purley) of London.

\section{Discussion and Conclusions}

Density as term and as measure is complex, fuzzy and delusive. While density is used in manifold studies for diverse purposes, our study quantitatively reveals that when using density measures a careful and clearly defined application is necessary:

First, the thematic dimension applied needs an unambiguous definition. When talking about density theoretically an uncountable amount of varying measures (people, jobs, built-up, etc.) could be used. Even as we reduced our understanding of density to a mere physical perspective on the city in this study, we are aware that the thematic variables used-building density, soil sealing, floor space density and the two generic structural types-must be seen as an incomplete proxy for the multidimensionality of the measure and for reality. The analysis of physical density of a city could be easily extended with parameters such as density of streets (by length, nodes, etc.), density of traffic, and density of office space, among many others for a more comprehensive picture. As an example, the street intersection density derived for London in 2010 by [20] reveals the area between the City and Hyde Park as the dominant center areas, which corresponds well with our results; however, 
the high density street intersection pattern extends to south of river Thames in their analysis; this is not reflected by our building density or floor space density pattern. Thus, in every analysis the thematic dimension must be understood as fragmentary for the density of the entire (physical) city system. Furthermore, the thematic definition of density is crucial as even related dimensions such as building density and floor space density are not linearly correlating $\left(r^{2}\right.$ of 0.66 in linear regression; $\mathrm{r}^{2}=0.42$ for soil sealing and building density; $\mathrm{r}^{2}=0.28$ for soil sealing and floor space density). Although there is a certain correlation measurable, these thematic objects are not interchangeable. It becomes clear that the usage of density in one way or the other needs a specific reference to the particular object. For example, the EUA data sets are criticized for hiding information on the urban morphology [47] and therefore pose an obstacle to the application in the domain of physical urban structure research; this is sometimes leading to a misunderstanding, when the density measures in the EUA are misinterpreted as building density instead of soil sealing.

Second, the spatial dimension needs an unambiguous definition. We approach this issue by: (a) applying a monocentric city model conceptualized by one defined center point; (b) applying a spatial concept using city scale and zonal scale as spatial baseline to account for city-wide and site-specific statements; and (c) using various reference units—block, grid and administrative units—to measure median and variance of density within the area of investigation. For a valid cross-city comparison, a consistent level for all spatial dimensions needs to be determined.

(a) The simplified conceptual monocentric city model applied for this study allows for comparative empirical tests to evaluate the density measure and its conceptual issues. However, numerous studies show that formerly monocentric cities transformed into ploycentric city configurations (e.g., [49,50]). Consequently, future studies on density measures should consider the particular structural configuration of the study sites.

(b) In our case, the city scale definition is data driven. However, this approach is exchangeable to any spatial unit, as long as the spatial baseline of the cities under test can be considered comparable. In turn, this implies that for cities featuring more complex extents (e.g., triggered by topographic situations) a challenge arises from defining appropriate and comparable spatial units beyond the simple ring model applied in this study.

(c) Furthermore, the spatial dimensions of applied reference units inherit significant impact on the results: Block and grid units show minor deviations regarding the relative distribution of density values; however, they determine the absolute density values significantly (grids lower the absolute measure by about $10 \%$ in our examples). Administrative units disqualify as reference unit, as their inherent delineation issues results in non-comparable numbers. An additional, but fundamental question is how appropriate grid and block units really are: It is obvious that the grids (the administrative units emphasize this effect) are at risk to spatially blur the real physical configuration by chance of their location (especially change-overs between high dense areas and open spaces level density values not representing either one of these structural type). The result shows that the grids consistently lower the absolute density values measured. Regarding the block units, the question remains, whether these units really meet their original intention: to capture homogeneous built-up structures. A general statement regarding this issue is also difficult due to the site specific nature of the analysis. Taubenböck et al. [51] delineated in their study homogeneous morphologic units via a data-driven approach using building density. Testing the influence of those homogeneous morphologic units relative to the block or gird units is suggested for future studies.

Third, the aggregation function has significant influence on the absolute measured density values and requires an unambiguous definition. On the one hand the calculation of gross or net densities is crucial. For example, we have demonstrated that net densities are naturally higher than gross densities; in our specific example the measured absolute values for gross densities are lower in the range of $4 \%$. 
On the other hand, when using generic structural classes, the population of data (drawing from all cities vs. one city at a time) is crucial.

Geographically the two areas of investigation-Paris and London-clearly reveal that the physical built-up configuration of Paris is significantly denser than in London. While this result is based on a systematic and clearly defined multidimensional analysis, less systematic approaches may lead to contradicting results. If we had calculated building density for both cities at a grid size of $35 \mathrm{~m}$, London would be in median denser than Paris. The conclusion London is denser then Paris would be logical; especially as the parameter is calculated for both cities at the same spatial extent, the same reference unit with the same calculation method and thus, in an ostensibly comparable way. Our systematic deconstruction of the measure density, however, lets us remark, this conclusion would be at least questionable due to its random selection of parameters. This example reveals how delusive density measures can be when based on random, unsystematic conceptions without sensitivity analyses. In contrast, for this specific case, all other grid sizes calculated confirm the original statement that Paris is denser than London. From another perspective on density measures, the statement Paris is denser than London is also misleading. If we take e.g., the parameter "density of open spaces" London has a higher density then Paris. Admittedly, open spaces are not intrinsically representing what the perception of density is. However, this example unmasks the complexity that comes with the use of various density measures. Moreover, the evaluation of the measure density of open spaces is justified as it also addresses the physical configuration of the city.

Beyond the specifically addressed research questions, this study has only become possible at a thematically and geometrically highly resolved level—even including the third dimension—due to recent advancement in geodata availability from remote sensing and VGI sources. We are aware that geodata derived from remote sensing and VGI are not cadastral data sets as they have to deal with accuracy issues. However, the accuracies of these data sets reveal consistency in themselves, typically having $80 \%-90 \%$ accuracy (e.g., [23,38]). Thus, we assume the influences of classification mistakes on the analysis of density measures are especially in a relative sense insignificant.

As concluding remark, this study reveals, whenever density is used as indicator, it is advisable to scrutinize the definition and calculation of density-with respect to the thematic and spatial dimensions as well as the calculus, and thus to verify the related interpretation. Current and past literature shows that an internationally accepted density measure is difficult to achieve as only a mixture out of a multitude of density perspectives may characterize the complex, multidimensional city configuration in a comprehensive and systematic way. This study systemizes conceptual issues and variables to be considered for transparent analyses, well-defined applications and meaningful interpretations. While we reduce our analysis to a physical approach of urban density, we argue that this concept can be seen as a blueprint for usage, understanding and interpretations for any other thematic approach such as population density.

\section{Outlook}

The expectations of global urban populations rising to $66 \%$ in 2050 , which equals additional 2.5 billion new city dwellers in the upcoming three decades [37], can be regarded an urban imperative to rethink which structure and morphology cities in the future can or should have. In spite of the dynamically growing amount of city dwellers (and the related dynamic spatial expansion of settlements (e.g., [52]), Angel et al. [31] discover a systematic decrease in density of global cityscapes in the twentieth century; that means, although the medial, cultural or scientific focus is often on high density vertical city centers or on high density informal settlements, horizontal urban growth with subsequent low densities (often described as "sprawl" (e.g., [53]) is the dominant global urban growth form. This implicitly has immense effects on available agricultural land, costs of public transport, ecologic footprints, etc. This development sets the tone for the need to better understand today's existing urban morphology and is a plea for intensified empirical evidence for the formation of informed opinions. 
As Hillier [54] stated the "spatial organization through buildings and built environments becomes one of the principle ways in which culture is made real for us in the material world, and it is because this is so that buildings can, and normally do, carry social ideas within their spatial forms". This emphasizes the need for systematically and consistently monitoring and analyzing the morphology of our cities on our planet, which is to date still done only in a very exemplary, often inconsistent way. This study supports this challenge by deconstructing the density measure and exemplifies its complexity and meaning along a comparative study between Paris and London. However, we do not evaluate density in normative terms. Instead we aim to add to the scientific debate giving a clear concept of how density measures can be defined, used, understood and which meaning they deliver. With it, we aim to approach a better documentation and thus understanding of the rigid physical structures of our cities, what was demanded e.g., by [55], as basis to better explain people's life situation.

Acknowledgments: The authors would like to specifically thank Prof. Dr. Peter Reinartz and Dr. Pablo D'Angelo for preprocessing and providing the Cartosat-1 nDSM data. We would also like to thank the three reviewers for their valuable comments that helped to significantly improve the manuscript.

Author Contributions: Hannes Taubenböck was responsible for the research design, the conceptual framework, the data analyses and wrote the initial manuscript. Ines Standfuß performed the data analyses and visualization, and contributed to the conceptual framework as well as to the written manuscript. Martin Klotzcollected the geodata and contributed to the conceptual framework as well as to the written manuscript. Michel Wurm processed the satellite dataand contributed to the conceptual framework as well as to the written manuscript. All authors discussed the results and commented the manuscript at all stages.

Conflicts of Interest: The authors declare no conflict of interest. The founding sponsors had no role in the design of the study, the data collection, the analyses, the interpretation of results, the process of writing of the manuscript or the decision to publish the results.

\section{References}

1. Jacobs, J. The Death and Life of Great American Cities; Random House: New York, NY, USA, 2016.

2. Burdett, R.; Travers, T.; Czischke, D.; Rode, P.; Moser, B. Density and Urban Neighborhoods in London; ISBN: 0-954888-1-1. Minerva LSE: London, UK, 2004.

3. Roskamm, N. Dichte: Eine transdisziplinäre Dekonstruktion. Diskurse zu Stadt und Raum. Reihe: Urban Studies; Transcript: Bielefeld, Germany, 2011. (In German)

4. Fina, S.; Krehl, A.; Siedentop, S.; Taubenböck, H.; Wurm, M. Dichter dran! Neue möglichkeiten der räumlichen analyse und visualisierung von stadtstrukturen mit dichteoberflächen und-profilen basierend aus erdbeobachtungsdaten. Raumforsch Raumordn 2014. [CrossRef]

5. Measuring Density: Working Definitions for Residential Density and Building Intensity. Available online: http:/ /www.corridordevelopment.org/pdfs/from_MDC_Website/db9.pdf (accessed on 16 November 2015).

6. INSEE: Populations legales 2012 de la commune. Available online: http://www.insee.fr/fr/ppp/basesde-donnees $/$ recensement $/$ populations-legales $/$ commune.asp?annee $=2012 \&$ depcom $=75056$ (accessed on 16 November 2015).

7. Openshaw, S. The Modifiable Areal Unit Problem. Norwick: Geo Books; ISBN: 0860941345. OCLC:12052482; GeoBooks: Norwhich, UK, 1983.

8. Weeks, J.R. Defining the urban area. In Remote Sensing of Urban and Suburban Area; Springer: Berlin, Germany, 2010.

9. Stahle, A. More Green Space in a Denser City—Critical Relations between User Experience and Urban Form; Springer: Berlin, Germany, 2008.

10. Sagl, G.; Resch, B.; Hawelka, B.; Beinat, E. From social sensor data to collective human behaviour patterns: Analyzing and visualizing spatio-temporal dynamics in urban environments. In Proceedings of the GI-Forum Geovisualization, Society and Learning, Salzburg, Austria, 3-6 July 2012.

11. Le Corbusier. Die Charte d' Athènes; Rowohlt Taschenbuchverlag: Hamburg, Germany, 1962. (In German)

12. Siedlungsentwicklung und Infrastrukturfolgekosten. Bilanzierung und Strategieentwicklung. Bonn. Available online: http://www.bbsr.bund.de/BBSR/DE/Veroeffentlichungen/BBSROnline/2002_2006/DL_ ON032006.pdf?_blob=publicationFile\&v=3 (accessed on 8 November 2016). (In German) 
13. McMillen, D.P. Employment densities, spatial autocorrelation \& subcenters in large metropolitan areas. J. Reg. Sci. 2004, 44, 225-243.

14. Glaeser, E. Triumph of the City; Penguin Press HC: London, UK, 2012.

15. Hoffmeyer-Zlotnik, J. Das "Wohnquartier" als Hintergrundmerkmal zur Erklärung von Umfragedaten. Festschrift für Max Kaase; Mohler, P. \& P. Luettinger (Hrsg.): Mannheim, Germany, 2000. (In German)

16. The Spatial Organization of Cities: Deliberate Outcome or Unforeseen Consequence? Available online: http:/ / escholarship.org/uc/item/5vb4w9wb\#page-4 (accessed on 16 November 2015).

17. Batty, M. The size, scale, and shape of cities. Science 2008, 319, 769-771. [CrossRef] [PubMed]

18. Barr, J.; Cohen, J.P. The floor area ratio gradient: New York City, 1890-2009. Reg. Sci. Urban Econom. 2014, 48, 110-119. [CrossRef]

19. Krehl, A.; Siedentop, S.; Taubenböck, H.; Wurm, M. A comprehensive view on urban spatial structure: Urban density patterns of German city regions. ISPRS Int. J. Geo-Inf. 2016. [CrossRef]

20. Masucci, A.P.; Stanilov, K.; Batty, M. Limited urban growth: London's street network dynamics since the 18th century. PLoS ONE 2013. [CrossRef] [PubMed]

21. Weng, Q. Remote sensing of impervious surfaces in the urban areas: Requirements, methods, and trends. Remote Sens. Environ. 2012, 117, 34-49. [CrossRef]

22. Anas, A.; Arnott, R.; Small, K. Urban spatial structure. J. Econom. Lit. 1998, 36, 1426-1464.

23. Wurm, M.; d'Angelo, P.; Reinartz, P.; Taubenböck, H. Investigating the applicability of Cartosat-1 DEMs and topographic maps to localize large-area urban mass concentrations. JSTARS 2014, 7, 4138-4152. [CrossRef]

24. Luck, M.; Wu, J. A gradient analysis of urban landscape pattern: A case study from the Phoenix metropolitan region, Arizona, USA. Landsc. Ecol. 2002, 17, 327-339. [CrossRef]

25. Guerois, M.; Pumain, D. Built-up encroachment and the urban field: A comparison of forty European cities. Environ. Plan. A 2008, 40, 2186-2203. [CrossRef]

26. McMillen, D.P. Testing for monocentricity. In A Companion to Urban Economics; Arnott, R.J., McMillen, D.P., Eds.; Blackwell Publishing: Malden, MA, USA, 2006; pp. 128-140.

27. Krehl, A. Urban spatial structure: An interaction between employment and built-up volumes. Reg. Stud. Reg. Sci. 2015, 2, 289-307. [CrossRef]

28. Poumadere, M.; Mays, C.; Le Mer, S.; Blong, R. The 2003 heat wave in France: Dangerous climate change here and now. Risk Anal. 2005, 25, 1483-1494. [CrossRef] [PubMed]

29. Wurm, M.; Taubenböck, H. Die Morphologie deutscher Großstädte: Was die Dichte über die Struktur der Städte verrät; Springer: Berlin, Germany, 2015. (In German)

30. Taubenböck, H.; Wurm, M. Wo beginnt die Stadt? Urbane Fernerkundung für stadtgeographische Forschung. DisP Plan. Rev. 2015, 3, 74-85. (In German)

31. The Persistent Decline in Urban Densities: Global and Historical Evidence of "Sprawl". Available online: https://www.lincolninst.edu/pubs/dl/1834_1085_Angel\%20Final\%201.pdf (accessed on 16 November 2015).

32. Banzhaf, E.; Höfer, R. Monitoring urban structure types as spatial indicators with CIR aerial photographs for a more effective urban environmental management. IEEE J. Sel. Topics Appl. Earth Obs. Remote Sens. 2008, 1, 129-138. [CrossRef]

33. Taubenböck, H.; Klotz, M.; Wurm, M.; Schmieder, J.; Wagner, B.; Wooster, M.; Esch, T. Central business districts: Delineation in mega city regions using remotely sensed data. Remote Sens. Environ. 2013, 136, 386-401.

34. Oliviera, V. Urban Morphology - An Introduction to the Study of the Physical Form of Cities; Springer: Berlin, Germany, 2016.

35. McFarlane, C. The geographies of urban density: Topology, politics and the city. Prog. Hum. Geogr. 2015, 40, 629-648. [CrossRef]

36. Westphal, C. Dichte und Schrumpfung. Kriterien zur Bestimmung angemessener Dichten in Wohnquartieren schrumpfender Städte aus Sicht der stadttechnischen Infrastruktur; Leibnitz-Institute für ökologische Raumentwickelung: Dresden, Germany, 2008. (In German)

37. United Nations (2014): World Urbanization Prospects-The 2014 Revision. Available online: http:/ / www. compassion.com/multimedia/world-urbanization-prospects.pdf (accessed on 4 November 2016).

38. UKMap Building Inventory. Available online: http://www.landmap.ac.uk/index.php/Datasets/Building Heights/Building-Heights-Download/menu-id-100339.html (accessed on 11 April 2012). 
39. OSM. 2015. Available online: http:/ /www.openstreetmap.de/ (accessed on 4 November 2016).

40. D'Angelo, P. Image matching and outlier removal for large scale DSM generation. In Proceedings of the ISPRS CGC \& ISPRS 2010, Calgary, AB, Canada, 15-18 June 2010.

41. EEA: Mapping Guide for a European Urban Atlas. Available online: http://www.eea.europa.eu/data-andmaps/data/urban-atlas/mapping-guide (accessed on 3 November 2016).

42. EEA: Urban Atlas. Available online: http://www.eea.europa.eu/data-and-maps/data/urban-atlas (accessed on 3 November 2016).

43. Eurostat. Available online: http://ec.europa.eu/eurostat/web/nuts/local-administrative-units (accessed on 3 November 2016).

44. Stahle, A. Compact sprawl-Exploring public open space and contradictions in urban density. In Proceedings of the 7th International Space Syntax Symposium, Stockholm, Sweden, 8-11 June 2008.

45. Batty, M.; Besussi, E.; Maat, K.; Harts, J. Representing multifunctional cities: Density and diversity in space and time. UCL Work. Paper Series 2003, 71. [CrossRef]

46. Everitt, B.S. The Cambridge Dictionary of Statistics, 2nd ed.; Cambridge: Cambridge, UK, 2002.

47. Bahrenberg, G.; Giese, E.; Nipper, J. Statistische Methoden in der Geographie. Vol 1, Univariate Statistik, Leipzig; Borntraeger: Stuttgart, Germany, 1999. (In German)

48. Prastacos, P.; Chrysoulakis, N.; Kochilakis, G. Urban Atlas, land use modelling and spatial metric techniques. In Proceedings of the 51st European Congress of the Regional Science Association International, Barcelona, Spain, 30 August-2 September 2011.

49. Siedentop, S. Ursachen, Ausprägungen und Wirkungen der globalen Urbanisierung-Ein Überblick. In Globale Urbanisierung - Perspektive aus dem All; Taubenböck, H., Wurm, M., Esch, T., Dech, S., Eds.; Springer: Berlin/Heidelberg, Germany, 2015; pp. 11-21. (In German)

50. Zhong, C.; Schläpfer, M.; Müller-Arisona, S.; Batty, M.; Ratti, C.; Schmitt, G. Revealing centrality in the spatial structure of cities from human activity patterns. Urban Stud. 2015. [CrossRef]

51. Taubenböck, H.; Habermeyer, M.; Roth, A.; Dech, S. Automated allocation of highly-structured urban areas in homogeneous zones from remote sensing data by Savitzky-Golay Filtering and curve sketching. IEEE Geosci. Remote Sens. Letters. 2006, 3, 532-536. [CrossRef]

52. Taubenböck, H.; Esch, T.; Felbier, A.; Wiesner, M.; Roth, A.; Dech, S. Monitoring of mega cities from space. Remote Sens. Environ. 2012, 117, 162-176. [CrossRef]

53. Siedentop, S. Urban sprawl-Verstehen, messen, steuern. Ansatzpunkte für ein empirisches Mess- und Evaluationskonzept der urbanen Siedlungsentwicklung. DisP Plan. Rev. 2005, 160, 23-35. [CrossRef]

54. Hillier, B. Space is the Machine; Cambridge University Press: Cambridge, UK, 1996.

55. Harvey, D. Justice, Nature E the Geography of Difference; Blackwell Publishers: Oxford, UK, 1996. 